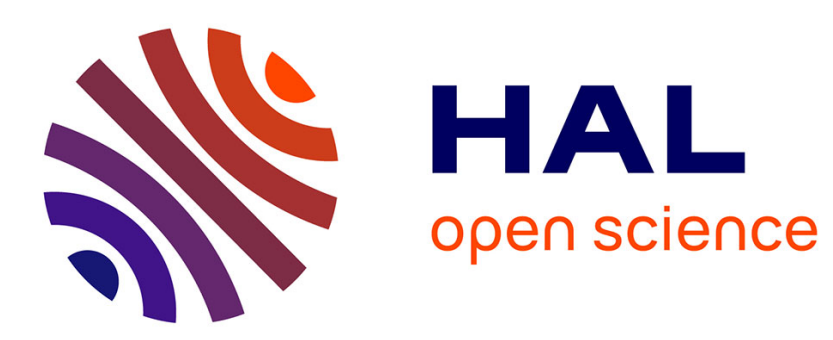

\title{
Extension of the destabilization paradox to limit cycle amplitudes for a nonlinear self-excited system subject to gyroscopic and circulatory actions
}

\author{
Benjamin Hervé, Jean-Jacques Sinou, Hervé Mahé, Louis Jezequel
}

\section{- To cite this version:}

Benjamin Hervé, Jean-Jacques Sinou, Hervé Mahé, Louis Jezequel. Extension of the destabilization paradox to limit cycle amplitudes for a nonlinear self-excited system subject to gyroscopic and circulatory actions. Journal of Sound and Vibration, 2009, 323 (3-5,), pp.944-973. 10.1016/j.jsv.2009.01.023 . hal-00411721

\author{
HAL Id: hal-00411721 \\ https://hal.science/hal-00411721
}

Submitted on 26 Sep 2012

HAL is a multi-disciplinary open access archive for the deposit and dissemination of scientific research documents, whether they are published or not. The documents may come from teaching and research institutions in France or abroad, or from public or private research centers.
L'archive ouverte pluridisciplinaire HAL, est destinée au dépôt et à la diffusion de documents scientifiques de niveau recherche, publiés ou non, émanant des établissements d'enseignement et de recherche français ou étrangers, des laboratoires publics ou privés. 


\title{
Extension of the Destabilization Paradox to Limit Cycle Amplitudes for a Nonlinear Self-Excited System subject to Gyroscopic and Circulatory actions
}

\author{
B. Hervé(1 and 2), J.-J. Sinou ${ }^{(2)}$, H. Mahé ${ }^{(1)}$ and L. Jézéquel ${ }^{(2)}$ \\ (1) Valeo Transmissions, Centre d'Étude des Produits Nouveaux, Espace Industriel Nord, Route de \\ Poulainville, 80009 Amiens Cedex 1, France \\ (2) Laboratoire de Tribologie et Dynamique des Systèmes UMR-CNRS 5513, École Centrale de Lyon, \\ 36 avenue Guy de Collongue, 69134 Écully Cedex, France \\ email: jean-jacques.sinou@ec-lyon.fr
}

\begin{abstract}
This study aims at clarifying the phenomenological roots of an acoustical disturbance known as "clutch squeal noise". A nonlinear two-degrees-of-freedom model is introduced in order to illustrate some basic phenomena leading to self-generated vibrations. The damping of the system as well as both circulatory and gyroscopic actions are included in order to highlight their respective influence and the destabilization paradox. Results are obtained on the stability range of the equilibrium, the nature of the Hopf bifurcation, the limit cycle branches and their stability. A dynamic extension of the destabilization paradox is proposed and some non-periodic behaviours are identified too.
\end{abstract}

Keywords: friction, destabilization paradox, limit cycles, self-generated vibrations

\section{Introduction}

In cars with manual transmissions, different unforced vibrations can be observed during the sliding phase of clutch engagement. Low frequencies phenomena such as judder can often be attributed to misalignment in the transmission chain or to tribological properties of the friction materials, such as a decreasing friction coefficient with regard to the sliding speed. However, noise due to high frequencies phenomena (up to few $\mathrm{kHz}$ ) can be experienced too and is referred as "clutch squeal noise". This audible disturbance can arise even when the friction coefficient is almost constant and the transmission chain shows no important fault. The observed amplitude of the phenomenon allows excluding the assumption of a stick-slip cycle too. The present study aims at investigating an instability origin of these vibrations related to the non-conservative action of the friction forces.

The non-conservative aspect of the friction forces is known to be a potential cause for the rise of such selfgenerated vibrations in dynamical systems through the destabilization of their stationary states. This has motivated numerous researchers to focus on such mechanisms for years, see [1]. Some other industrial applications in need of such studies can be cited without being exhaustive: brakes, machining, civil engineering and aeronautic design.

In general, a stability analysis only requires the knowledge of the linearized equations of motion in the vicinity of the equilibriums, see [2]. Therefore, phenomenological roots of the spontaneous rise of motion can be highlighted through rather simple considerations. However, the price to pay for this simplicity is the complete ignorance of the nature and of any property of the steady state which then takes place. This lack of knowledge can lead to misinterpretation of experimental observations as well as bad or excessively stringent design recommendations.

The present paper aims at demonstrating the important role of the nonlinearities and the damping structure in dynamical systems subject to flutter destabilization. To this purpose, a paradigmatic example 
model of non-conservative coupling between the degrees of freedom of a clutch is proposed in the first part. This nonlinear model shows two degrees of freedom coupled by both circulatory and gyroscopic actions and takes into account the structural damping of the system.

The second part serves as a reminder about fundamental results on the stability of the stationary states. Considerations for the damping structure of the system lead to counter-intuitive results and the so-called "destabilization paradox". This phenomenon was first reported by Ziegler in [3]: he demonstrated the significance of dynamic terms and the destabilizing effect of weak damping for a double pendulum subjected to tangential load. Further developments were proposed by Bolotin [4] for non-conservative stability problems. Then, some researchers (Hermann et al. [6], Leipholz [5] and Kounadis [22]) studied the destabilizing effect of viscous damping for mechanical systems subjected to non-conservative forces. Moreover, Thomsen [20] examined the non-linear dynamics of a double pendulum with both linear damping and non-conservative follower loading. He studied the occurrence of chaotic motion and changes in amplitude due to a destabilizing effect of both linear and non-linear forces. The influence of the velocity-dependent forces on the stability of non-conservative systems as well as the effects of non-linearities were also studied by O'Reilly et al. [7] and Kirillov and Seyranian [8]. In spite of these intensive investigations, this topic still motivates researches aiming at understanding the complex destabilization mechanisms in presence of damping, see for example [9-13].

A static influence of the nonlinearities is also evocated in the determination of the equilibriums and the nature of the Hopf bifurcation points by the analysis of the first Lyapounov coefficient. The important role of the structural damping is clearly illustrated and a particular relationship to the gyroscopic action is highlighted.

The third part introduces a nonlinear method for determining the limit cycles of autonomous mechanical systems which is appropriate to perform parametric studies. An application of the Floquet theory is also described in order to determine the stability nature of the identified periodic solutions. Then, this approach is applied on the clutch model to investigate its limit cycles. The complex dynamic behaviour of nonlinear structures is clearly illustrated on this example. Neimark-Sacker bifurcations and a possible route to chaos according to the Ruelle-Takens scenario are highlighted with the help of Poincaré sections. Such a turbulent behaviour was already mentioned in [20] for instance.

Finally, conclusions are drawn on the sensitivity of self-generated vibrations in mechanical structures to the physical parameters. A particular attention is paid to the role of the structural damping and the gyroscopic actions both on the stability of the equilibriums and the post-bifurcation dynamic behaviour.

\section{2-DOF nonlinear model of a squealing clutch}

The proposed model of clutch is depicted on Fig. 1. This system was first proposed by Wickramarachi in [14] and extensively investigated in the proposed simplified form depicted on Fig. 1 by the authors in [13]. It consists of two discs $(A)$ and $(B)$ coaxially rotating about the $O \mathbf{z}$ axis and rubbing on each other. $O x y z$ is the principal inertia frame of $(A) .(A)$ is the friction disc and $(B)$ represents the engine flywheel and the clutch.

Two degrees of freedom (DOF) are considered. They correspond to the swinging motions of $(A)$. The swinging angles about $O \mathbf{x}$ and $O \mathbf{y}$ are noted $\theta$ and $\phi$ respectively. Restoring forces about these motions are represented by linear stiffness and damping elements, $\left(k_{\theta}, d_{\theta}\right)$ and $\left(k_{\phi}, d_{\phi}\right)$ about $O \mathbf{x}$ and $O \mathbf{y}$ respectively. Friction is modeled in a simplified manner: four deformable elements are considered, equally distributed on radius $R$ on $(A)$, and friction occurs at their end. They represent the flexibility of the contact area and they are assumed to show equal linear stiffness and damping $\left(k_{c}, d_{c}\right)$ about $O \mathbf{x}$. A constant Coulomb law is considered with the constant friction coefficient $\mu$. The rotation speed of both the discs is assumed to be constant. The parallelism of the discs $(\theta=0$ and $\phi=0)$ illustrated on Fig. 1 is 


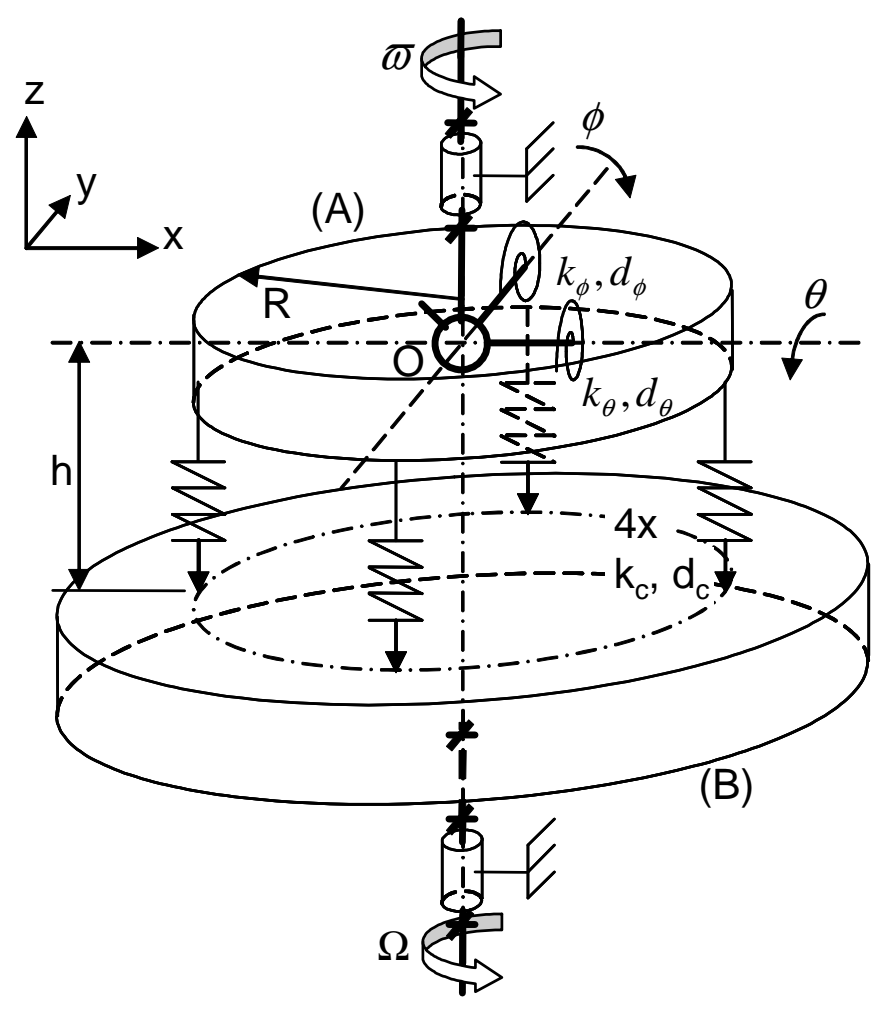

Figure 1: Model of rubbing discs subject to a flutter destabilization

supposed to correspond to equilibrium. The distance between the $O \mathbf{x y}$ plane and the end of the contact elements is noted $h$. Finally, the amplitude of the vibrations is assumed to be small enough so that the friction force at the end of each contact element never vanishes.

The following nonlinear nondimensional equation of motion can be obtained for this system,

$$
\left[\begin{array}{c}
\ddot{\theta} \\
\ddot{\phi}
\end{array}\right]+\left[\begin{array}{cc}
2 \xi & \rho \\
-\rho & 2 \alpha \beta \xi
\end{array}\right]\left[\begin{array}{c}
\dot{\theta} \\
\dot{\phi}
\end{array}\right]+\left[\begin{array}{cc}
1 & \varphi \\
-\varphi & \alpha^{2}
\end{array}\right]\left[\begin{array}{l}
\theta \\
\phi
\end{array}\right]+\sigma\left[\begin{array}{l}
\theta^{3} \\
\phi^{3}
\end{array}\right]=\left[\begin{array}{l}
0 \\
0
\end{array}\right] .
$$

The upper dot denotes time differentiation with regard to a nondimensional time scale $\tau$. $\xi \geq 0$ is the reference damping factor. $\alpha \geq 1$ and $\beta \geq 0$ are the ratios of the natural frequencies and damping factors respectively. $\varphi$ and $\rho$ are the nondimensional circulatory and gyroscopic factors respectively. Finally, $\sigma>0$ is related to the local stiffening property of the system in the case of nonvanishing motions.

These nondimensional parameters are related to the physical parameters with the expression reported in 
Eq. 2

$$
\left\{\begin{array}{l}
\omega_{0}=\sqrt{\frac{1}{J}\left(2 R^{2} k_{c}+k_{\theta}-\frac{N_{0}}{2} h\left(2+\mu^{2}\right)+\left(J_{\varpi}-J\right) \varpi^{2}\right)} \\
\xi=\frac{1}{2 \omega_{0}} \frac{1}{J}\left(2 R^{2} d_{c}+d_{\theta}+\mu \frac{N_{0}}{2} \frac{h^{2}}{R|\varpi-\Omega|}\right) \\
\rho=\frac{1}{\omega_{0}} \frac{1}{J}\left(2 \mu R h d_{c} \operatorname{sign}(\varpi-\Omega)+\left(J_{\varpi}-2 J\right) \varpi\right) \\
\varphi=-\frac{1}{\omega_{0}^{2}} \frac{1}{J} \mu R\left(\frac{N_{0}}{2}\left(1+\frac{h^{2}}{R^{2}}\right)-2 h k_{c}\right) \operatorname{sign}(\varpi-\Omega) \\
\alpha=\frac{1}{\omega_{0}} \sqrt{\frac{1}{J}\left(2 R^{2} k_{c}+k_{\phi}-\frac{N_{0}}{2} h\left(2+\mu^{2}\right)+\left(J_{\varpi}-J\right) \varpi^{2}\right)} \\
\beta=\frac{1}{\xi} \frac{1}{2 \alpha \omega_{0}} \frac{1}{J}\left(2 R^{2} d_{c}+d_{\phi}+\mu \frac{N_{0}}{2} \frac{h^{2}}{R|\varpi-\Omega|}\right)
\end{array},\right.
$$

where $t$ is the natural time scale, $J$ is the swinging inertia about $O \mathbf{x}$ and $O \mathbf{y}, J_{\varpi}$ is the rotational inertia about $O \mathbf{z}, \varpi$ is the rotation speed of $(A), \Omega$ is the rotation speed of $(B)$ and $N_{0}$ is the load applied to the system.

A particular symmetry of Eq. 1 can be observed since if $\left[\begin{array}{ll}\theta & \phi\end{array}\right]^{T}$ is a solution for a given value of $(\varphi, \rho)=\left(\varphi_{0}, \rho_{0}\right)$ then $\left[\begin{array}{ll}\theta & -\phi\end{array}\right]^{T}$ is a solution for $(\varphi, \rho)=\left(-\varphi_{0},-\rho_{0}\right)$. Therefore, the investigation of negative values of $\varphi$ can be omitted in any parametric investigation. One can also note that the amplitude of $\sigma$ has no qualitative effect on the solutions since if $\left[\begin{array}{ll}\theta & \phi\end{array}\right]^{T}$ is a solution for $\sigma=\sigma_{0}$ then $\sqrt{\sigma_{0} / \sigma}\left[\begin{array}{ll}\theta & \phi\end{array}\right]^{T}$ is a solution for any other value of $\sigma$.

$\left[\begin{array}{ll}0 & 0\end{array}\right]^{T}$ is the unique equilibrium of Eq. 1. Finally, the linearization of Eq. 1 in the vicinity of the equilibrium simply gives

$$
\left[\begin{array}{l}
\ddot{\theta} \\
\ddot{\phi}
\end{array}\right]+\left[\begin{array}{cc}
2 \xi & \rho \\
-\rho & 2 \alpha \beta \xi
\end{array}\right]\left[\begin{array}{c}
\dot{\theta} \\
\dot{\phi}
\end{array}\right]+\left[\begin{array}{cc}
1 & \varphi \\
-\varphi & \alpha^{2}
\end{array}\right]\left[\begin{array}{l}
\theta \\
\phi
\end{array}\right]=\left[\begin{array}{l}
0 \\
0
\end{array}\right] .
$$

The values used for numerical illustrations in this paper are $\alpha=1.5$ and $\sigma=10^{4}$. As highlighted in [13] the value of $\alpha$ has no qualitative effect on the stability analysis, provided $\alpha \neq 1$. Therefore, the choice $\alpha=1.5$ is representative of any assumed difference between the static frequencies of the swinging modes for the stability analysis. Moreover, for a fixed value of $\alpha$, the value of $\sigma$ has no qualitative effect neither, as mentioned later. The choice of $\sigma=10^{4}$ allows obtaining reasonable amplitudes for the limit cycles. The influence of the values of both $\alpha$ and $\sigma$ together on the nonlinear behaviour is not analyzed in this paper. Parametric investigations are performed on the other parameters.

\section{Investigation of equilibriums}

The equilibriums are steady static states of a system. Their investigation aims at understanding the reasons why a dynamic state arises preferentially to equilibrium. This section is divided into three parts. Firstly, the eigenvalues approach of the stability analysis is introduced in order to highlight how equilibrium can become a repulsive state. Secondly, a local insight into the nonlinear properties of the system in the vicinity of bifurcations is proposed in order to describe the loss of stability and explain jump phenomena, i.e. the sudden rise of a large motion when crossing the stability frontier. Finally, the stability of the equilibrium of the proposed model is investigated and important information is obtained on the role of damping and gyroscopic actions. 


\subsection{Stability analysis}

Consider the equation of motion of a dynamic system

$$
\dot{\mathbf{X}}=\mathbf{f}(\mathbf{X}, \mathbf{p}),
$$

where $\mathbf{X}$ represents the instantaneous state of the system (its coordinates in the phase space), the upper dot denotes time derivation and $\mathbf{f}$ is a function of $\mathbf{X}$ parameterized by the elements of $\mathbf{p}$. Assume at least one stationary solution $\mathbf{X}_{e}\left(\mathbf{p}_{0}\right)$ exists for $\mathbf{p}=\mathbf{p}_{0}$, i.e. $\mathbf{f}\left(\mathbf{X}_{e}\left(\mathbf{p}_{0}\right), \mathbf{p}_{0}\right)=\mathbf{0}$, and $\mathbf{f}$ is $C^{1}$ in its vicinity. Then, according to the Hartman-Grobman theorem, the linearization of Eq. 4 in the vicinity of $\mathbf{X}_{e}\left(\mathbf{p}_{0}\right)$ preserves its non-marginal stability nature. Therefore, the determination of the stability nature of equilibriums only requires the knowledge of the linearized equation of motion in their vicinity in most cases.

Nevertheless, the nonlinearities allow many equilibriums to exist simultaneously and their evolution when varying $\mathbf{p}$ can be rather complex and possibly discontinuous. Therefore, all equilibrium branches have to be determined by appropriate approaches as a first step prior to perform a stability analysis of each branch separately.

Assume the linearized form of Eq. 4 expressed in Eq. 5,

$$
\dot{\mathbf{X}} \approx \mathrm{D} \mathbf{f}\left(\mathbf{X}_{e}\left(\mathbf{p}_{0}\right), \mathbf{p}_{0}\right) \mathbf{X}
$$

The solutions of such a linear system form a vector space whose basis is constituted of elements of the form expressed in Eq. 6 when the Jacobian is diagonalisable, where $\mathbf{X}_{0}\left(\mathbf{p}_{0}\right)$ is the mode shape, $t$ the time and $\lambda\left(\mathbf{p}_{0}\right)$ a complex factor. $\lambda\left(\mathbf{p}_{0}\right)$ and $\mathbf{X}_{0}\left(\mathbf{p}_{0}\right)$ are an eigenvalue and an associated eigenvector of $\mathrm{D} \mathbf{f}\left(\mathbf{X}_{e}\left(\mathbf{p}_{0}\right), \mathbf{p}_{0}\right)$.

$$
\mathbf{X}(t)=\mathbf{X}_{0}\left(\mathbf{p}_{0}\right) \mathrm{e}^{\lambda\left(\mathbf{p}_{0}\right) t} .
$$

Because of the form of the solutions, the stability nature of $\mathbf{X}_{e}\left(\mathbf{p}_{0}\right)$ is expressed by the eigenvalues of the Jacobian D $\mathbf{f}\left(\mathbf{X}_{e}\left(\mathbf{p}_{0}\right), \mathbf{p}_{0}\right)$. Assume the Jacobian has no purely imaginary eigenvalue (hyperbolic equilibrium). If all the eigenvalues show a strictly negative real part then the equilibrium is asymptotically stable; if at least one eigenvalue shows a strictly positive real part then the equilibrium is unstable. The imaginary part indicates the oscillatory or non-oscillatory nature of the corresponding mode.

In the case the Jacobian is not diagonalisable, the solutions have the more general form expressed in Eq. 7 , where $\mathbf{P}$ is a polynomial whose order is strictly lower than the multiplicity order of the associated eigenvalue $\lambda$.

$$
\mathbf{X}(t)=\mathbf{P}\left(t, \mathbf{p}_{0}\right) \mathrm{e}^{\lambda\left(\mathbf{p}_{0}\right) t} .
$$

This situation can only lead to instability if the real part of at least one eigenvalue is strictly positive or if the Jacobian has at least one purely imaginary eigenvalue.

The existence of purely imaginary eigenvalues lacks of robustness with regard to physical parameters. However, the sign of the real part of the eigenvalues is a robust criterion. Furthermore, because of the continuity of the eigenvalues with regard to the elements of $\mathrm{D} \mathbf{f}\left(\mathbf{X}_{e}\left(\mathbf{p}_{0}\right), \mathbf{p}_{0}\right)$, the locus of purely imaginary eigenvalues in the parameters space form continuous surfaces, provided the equilibrium evolves continuously with $\mathbf{p}$. These surfaces separate regions of asymptotic stability and instability, thus they constitute stability frontiers.

As reported in many previous works the damping structure has an important influence on the stability frontiers. In [9], Kirillov considers 2-DOF purely circulatory and purely gyroscopic undamped systems. He demonstrates for both these systems that the limit of the domain of asymptotic stability as the amplitude of a perturbing action vanishes can differ from the domain of marginal stability of the unperturbed systems, this being referred as the destabilization paradox. As a consequence, significant differences can be found between experienced and theoretically predicted stability frontiers. This effect emphasizes the 
strong influence of the structure of the system on the stability frontiers independently from the amplitude of the parameters.

\subsection{Nature of the Hopf bifurcation}

Assume the system described by Eq. 4 shows a Hopf bifurcation for $\mathbf{p}=\mathbf{p}_{0}+\Delta \mathbf{p}$ with regard to the element $p_{i}$ of $\mathbf{p}$. By this is meant that $\mathbf{f}$ is assumed to be regular with regard to $\mathbf{X}$ and $p_{i}$ at $\left(\mathbf{X}_{e}\left(\mathbf{p}_{0}\right), \mathbf{p}_{0}\right)$, $\mathrm{D} \mathbf{f}\left(\mathbf{X}_{e}\left(\mathbf{p}_{0}\right), \mathbf{p}_{0}\right)$ shows one pair of purely imaginary eigenvalues $\lambda_{1,2}\left(\mathbf{p}_{0}\right)= \pm i \omega_{0}$ with $\omega_{0}>0$, all the other eigenvalues show a strictly negative real part and

$$
\frac{\mathrm{d} \operatorname{Re}\left(\lambda_{1,2}\right)}{\mathrm{d} p_{i}}\left(\mathbf{p}_{0}\right) \neq 0
$$

This situation corresponds to the crossing of the stability frontier and the transition between an asymptotically stable and an unstable nature of the equilibrium. Without loss of generality, one can assume $\mathbf{p}_{0}=\mathbf{0}$ and $\mathbf{X}_{e}(\mathbf{p})=\mathbf{0}$. For $\left|p_{i}\right|$ sufficiently small, let $\mathbf{X}_{0}(\mathbf{p})$ be associated to $\lambda_{1}(\mathbf{p})$ and $\mathbf{X}_{0}^{*}(\mathbf{p})$ be its adjoint eigenvector, i.e. $\mathrm{D} \mathbf{f}\left(\mathbf{X}_{e}(\mathbf{p}), \mathbf{p}\right) \mathbf{X}_{0}(\mathbf{p})=\lambda_{1}(\mathbf{p}) \mathbf{X}_{0}(\mathbf{p})$ and $\operatorname{Df}\left(\mathbf{X}_{e}(\mathbf{p}), \mathbf{p}\right)^{T} \mathbf{X}_{0}^{*}(\mathbf{p})=$ $\overline{\lambda_{1}}(\mathbf{p}) \mathbf{X}_{0}^{*}(\mathbf{p})$, such that $\left\langle\mathbf{X}_{0}(\mathbf{p})^{*}, \mathbf{X}_{0}(\mathbf{p})\right\rangle=1$ where $\langle\bullet, \bullet\rangle$ is the inner product $\left\langle\left[u_{i}\right],\left[v_{i}\right]\right\rangle=\sum_{i} \bar{u}_{i} v_{i}$. According to the Shoshitaishvili theorem the topology of Eq. 4 in the vicinity of $\mathbf{p}_{0}$ can be judged from its restriction to a local center manifold, expressed as

$$
\mathbf{X}(t)=\varsigma(t) \mathbf{X}_{0}(\mathbf{p})+\bar{\varsigma}(t) \overline{\mathbf{X}_{0}}(\mathbf{p})+\sum_{i+j=2}^{3} \frac{1}{i ! j !} \mathbf{a}_{i j}(\mathbf{p}) \varsigma^{i} \bar{\varsigma}^{j}+O\left(|\varsigma|^{4}\right),
$$

which is locally topologically equivalent to the restriction

$$
\mathbf{X}(t)=q(t) \mathbf{X}_{0}(\mathbf{p})+\bar{q}(t) \overline{\mathbf{X}_{0}}(\mathbf{p}), \text { with } q(t)=\left\langle\mathbf{X}_{0}^{*}(\mathbf{p}), \mathbf{X}(t)\right\rangle .
$$

Eq. 4 can be changed into Eq. 11,

$$
\dot{\mathbf{X}}=\mathrm{D} \mathbf{f}\left(\mathbf{X}_{e}(\mathbf{p}), \mathbf{p}\right) \mathbf{X}+\mathbf{g}(\mathbf{X}, \mathbf{p}) \text {, with } \mathbf{g}=\mathrm{O}\left(\|\mathbf{X}\|^{2}\right) .
$$

Then, it follows from Eq. 10 that

$$
\dot{q}=\lambda_{1}(\mathbf{p}) q+\left\langle\mathbf{X}_{0}^{*}(\mathbf{p}), \mathbf{g}\left(q \mathbf{X}_{0}(\mathbf{p})+\bar{q} \overline{\mathbf{X}_{0}}(\mathbf{p}), \mathbf{p}\right)\right\rangle .
$$

Provided $\mathbf{f}$ is regular enough, a polynomial expansion can be proposed, see Eq. 13,

$$
\dot{q}=\lambda_{1}(\mathbf{p}) q+\sum_{i+j=2}^{3} \frac{1}{i ! j !} b_{i j}(\mathbf{p}) q^{i} \bar{q}^{j}+\mathrm{O}\left(|q|^{4}\right),
$$

with

$$
b_{i j}(\mathbf{p})=\frac{\partial^{i+j}}{\partial q^{i} \partial \bar{q}^{j}}\left\langle\mathbf{X}_{0}^{*}(\mathbf{p}), \mathbf{g}\left(q \mathbf{X}_{0}(\mathbf{p})+\bar{q} \overline{\mathbf{X}_{0}}(\mathbf{p}), \mathbf{p}\right)\right\rangle(q=0) .
$$

Then, the Poincaré normal form Eq. 16 is obtained by considering a pertinent change of variable in the form of Eq. 15,

$$
\begin{gathered}
q=r+\sum_{i+j=2}^{3} \frac{1}{i ! j !} h_{i j}(\mathbf{p}) r^{i} \bar{r}^{j}, \\
\dot{r}=\lambda_{1}(\mathbf{p}) r+\gamma(\mathbf{p})|r|^{2} r+O\left(|r|^{4}\right),
\end{gathered}
$$




$$
\gamma(\mathbf{p})=\frac{2 \lambda_{1}(\mathbf{p})+\overline{\lambda_{1}}(\mathbf{p})}{2\left|\lambda_{1}(\mathbf{p})\right|^{2}} b_{11} b_{20}+\frac{\left|b_{11}\right|^{2}}{\lambda_{1}(\mathbf{p})}+\frac{\left|b_{02}\right|^{2}}{2\left(2 \lambda_{1}(\mathbf{p})-\overline{\lambda_{1}}(\mathbf{p})\right)}+\frac{b_{21}}{2} .
$$

Finally, a change of variable and time scale expressed in Eq. 18 allows writing Eq. 19 for $\left|p_{i}\right|$ sufficiently small,

$$
\begin{gathered}
r=\left|\frac{\operatorname{Re}(\gamma(\mathbf{p}))}{\operatorname{Im}\left(\lambda_{1}(\mathbf{p})\right)}-\frac{\operatorname{Re}\left(\lambda_{1}(\mathbf{p})\right) \operatorname{Im}(\gamma(\mathbf{p}))}{\operatorname{Im}^{2}\left(\lambda_{1}(\mathbf{p})\right)}\right|^{-1 / 2} s \text { and } \mathrm{d} \tau=\frac{1}{\omega_{0}}\left(1-\operatorname{Im}\left(\frac{\gamma(\mathbf{p})}{\omega_{0}}\right)|r|^{2}\right) \mathrm{d} t, \\
\frac{\mathrm{d} s}{\mathrm{~d} \tau}=\left(\frac{\operatorname{Re}\left(\lambda_{1}(\mathbf{p})\right)}{\operatorname{Im}\left(\lambda_{1}(\mathbf{p})\right)}+i\right) s+\operatorname{sign}(\operatorname{Re}(\gamma(\mathbf{0})))|s|^{2} s+O\left(|s|^{4}\right) .
\end{gathered}
$$

According to the center manifold theory, the dynamical behaviour of Eq. 4 is directly related to the behaviour of Eq. 20 in the vicinity of the Hopf bifurcation,

$$
\begin{gathered}
\frac{\mathrm{d} s}{\mathrm{~d} \tau}=\left(\frac{\operatorname{Re}\left(\lambda_{1}(\mathbf{p})\right)}{\operatorname{Im}\left(\lambda_{1}(\mathbf{p})\right)}+i\right) s+\operatorname{sign}\left(l_{1}\right)|s|^{2} s, \\
l_{1}=\operatorname{Re}\left(i b_{11} b_{20}+\omega_{0} b_{21}\right) .
\end{gathered}
$$

$l_{1}$ defined in Eq. 21 is the first Lyapunov coefficient. Assume $l_{1} \neq 0$; it can be seen from Eq. 20 that a limit cycle branch connects with the equilibrium branch at the Hopf bifurcation point. This is why the Hopf bifurcation is an appropriate candidate phenomenon to explain the rise of self-generated vibrations in dynamical systems. The stable or unstable nature of this limit cycle, related to the supercritical or subcritical nature of the bifurcation respectively, can be judged from the sign of $l_{1}$. If $l_{1}<0$ (respectively $l_{1}>0$ ) then the bifurcation is supercritical (respectively subcritical). In the case of a degenerated Hopf bifurcation $\left(l_{1}=0\right)$ further developments can be proposed to determine the next Lyapunov coefficients. The interested reader is referred to [16] and [17].

The determination of the nature of a Hopf bifurcation is of particular importance when dealing with the suppression of self-generated vibrations caused by such a mechanism. Indeed, a supercritical bifurcation allows reducing the amplitude of the limit cycle as much as desired in the vicinity of the stability frontier. Therefore, an acceptable level can be reached without stabilizing the equilibrium. Contrariwise, a subcritical bifurcation leads to a jump phenomenon at the stability frontier between the equilibrium branch and the attracting steady state. Moreover, stable vibrations can remain even after the equilibrium is stabilized.

Assume a polynomial expression of $\mathbf{g}$ in Eq. 11 in the form of Eq. 22,

$$
\mathbf{g}(\mathbf{X}, \mathbf{p})=\frac{1}{2} \mathbf{P}(\mathbf{X}, \mathbf{X}, \mathbf{p})+\frac{1}{6} \mathbf{Q}(\mathbf{X}, \mathbf{X}, \mathbf{X}, \mathbf{p})+O\left(|\mathbf{X}|^{4}\right),
$$

with

$$
\mathbf{P}(\mathbf{U}, \mathbf{V}, \mathbf{p})=\sum_{i, j \geqslant 1} \frac{\partial^{2} \mathbf{g}(\mathbf{X}, \mathbf{p})}{\partial x_{i} \partial x_{j}} u_{i} v_{j}(\mathbf{X}=0)
$$

and

$$
\mathbf{Q}(\mathbf{U}, \mathbf{V}, \mathbf{W}, \mathbf{p})=\sum_{i, j, k \geqslant 1} \frac{\partial^{3} \mathbf{g}(\mathbf{X}, \mathbf{p})}{\partial x_{i} \partial x_{j} \partial x_{k}} u_{i} v_{j} w_{k}(\mathbf{X}=0)
$$

where $x_{i}$ is the $i$-th component of $\mathbf{X}$. Because of the relation between Eq. 4 and Eq. 16 above mentioned, vectors $\mathbf{a}_{\mathbf{i j}}(\mathbf{p})$ can be directly identified in Eq. 9 so that

$$
\dot{\varsigma}=\lambda_{1}(\mathbf{p}) \varsigma+\frac{1}{2} \Gamma(\mathbf{p})|\varsigma|^{2} \varsigma+\mathrm{O}\left(|\varsigma|^{4}\right) .
$$


Writing $\Gamma, \mathbf{X}_{0}, \mathbf{P}(\mathbf{U}, \mathbf{V}), \mathbf{Q}(\mathbf{U}, \mathbf{V}, \mathbf{W})$ and $\mathrm{D} \mathbf{f}$ for $\Gamma(0), \mathbf{X}_{0}(0), \mathbf{P}(\mathbf{U}, \mathbf{V}, \mathbf{0}), \mathbf{Q}(\mathbf{U}, \mathbf{V}, \mathbf{W}, \mathbf{0})$ and $\mathrm{D} \mathbf{f}(\mathbf{0}, \mathbf{0})$ respectively, substituting Eq. 9 into Eq. 4 and identifying the vectors $\mathbf{a}_{i j}(\mathbf{p})$ in order to fit the form of Eq. 25 for $\mathbf{p}=\mathbf{0}$ one obtains the following criterion for the nature of the Hopf bifurcation,

$$
\begin{gathered}
L_{1}=\operatorname{Re}(\Gamma), \\
\Gamma=\left\langle\mathbf{X}_{0}^{*}, \mathbf{Q}\left(\mathbf{X}_{0}, \mathbf{X}_{0}, \overline{\mathbf{X}_{0}}\right)+\mathbf{P}\left(\overline{\mathbf{X}_{0}},\left(2 i \omega_{0} \mathbf{I}-D \mathbf{f}\right)^{-1} \mathbf{P}\left(\mathbf{X}_{0}, \mathbf{X}_{0}\right)\right)-2 \mathbf{P}\left(\mathbf{X}_{0}, D \mathbf{f}^{-1} \mathbf{P}\left(\mathbf{X}_{0}, \overline{\mathbf{X}_{0}}\right)\right)\right\rangle
\end{gathered}
$$

where $\mathbf{I}$ is the identity matrix. Of course, the criterion on the sign of $L_{1}$ defined in Eq. 26 and the sign of $l_{1}$ defined in Eq. 21 are equivalent, but the latter expression can be more convenient when an expression of $\mathbf{g}$ in the form of Eq. 22 is available.

\subsection{Stability analysis of the squealing clutch}

A complete parametric analysis of the modes and the stability domain of the equilibrium was proposed by the authors in [13]. Eq. 3 can be changed to fit the form of Eq. 4 by considering the state variable $\mathbf{X}=\left[\begin{array}{llll}\theta & \phi & \dot{\theta} & \dot{\phi}\end{array}\right]^{T}$.

Provided no degree of freedom is over-damped, the characteristic polynomial of the Eq. 3 is strictly positive on the real axis. Therefore, the eigenvalues of the system can not be real. Thus, the trivial equilibrium can only destabilize by flutter. In order to distinguish the effect of the damping amount and its distribution over the two coupled degrees of freedom, one calls $\xi_{t}=\xi(1+\alpha \beta)$ the total damping amount. Then, for $\xi_{t} \neq 0$ the stability frontier is described by (see [13])

$$
\left(\varphi \rho+\alpha \xi_{t}\left(\frac{\alpha+\beta}{1+\alpha \beta}\right)\right)^{2}-\xi_{t}\left(\varphi \rho+\alpha \xi_{t} \frac{\alpha+\beta}{1+\alpha \beta}\right)\left(1+\alpha^{2}+\rho^{2}+4 \frac{\alpha \beta}{(1+\alpha \beta)^{2}} \xi_{t}^{2}\right)+\xi_{t}^{2}\left(\alpha^{2}+\varphi^{2}\right)=0,
$$

The frontiers for $\alpha=1.5$ are depicted on Fig. 2 and 3 for various iso-distributed damping amounts and for various damping distributions respectively. The domain of marginal stability of the associated undamped system has also been reported. On this figure, stability is found in the central area and the equilibrium experiences a Hopf bifurcation when the parameters cross the frontiers.

It can be shown that the illustrated behaviour is not qualitatively affected by the value neither of $\alpha$ nor $\xi_{t}$ provided $\alpha \neq 1, \xi_{t} \neq 0$ and $\xi_{t}$ sufficiently small. For $\alpha=1$ the domain of marginal stability of the undamped purely circulatory system collapses and this also affects the frontiers of the damped system. However, this situation physically corresponds to the equality of the frequencies of two distinct modes and is unlikely to occur in a real system including some geometrical defects. Therefore Fig. 2 and 3 correctly illustrate the respective influence of the parameters in the general case.

An important observation concerns the distinct role of the damping amount and its distribution respectively. On the one hand, the presence of damping adds robustness to stability by changing the domain of marginal stability into a domain of asymptotic stability whose size depends on the damping amount. On the other hand, the damping structure rules the shape of the stability frontiers.

This contrast can be emphasized in the manner of Kirillov [9] by considering a vanishing perturbation of an undamped system. As an example, assume a constant structure of the damping matrix with a fixed value of $\beta$ and $\rho=\eta \xi_{t}$, i.e. a perturbed undamped purely circulatory system. Then, as $\xi_{t}$ vanishes, Eq. 28 tends to

$$
(1+\alpha \beta)^{2}\left(1+\eta^{2}\right) \varphi^{2}+\left(\alpha^{2}-1\right)\left(1-\alpha^{2} \beta^{2}\right) \eta \varphi-\alpha \beta\left(\alpha^{2}-1\right)^{2}=0 .
$$

The limits of the stability frontiers as the perturbation vanishes are reported on Fig. 4. It is obvious from this expression that the stability frontiers of the vanishingly damped system do not necessarily tend to 


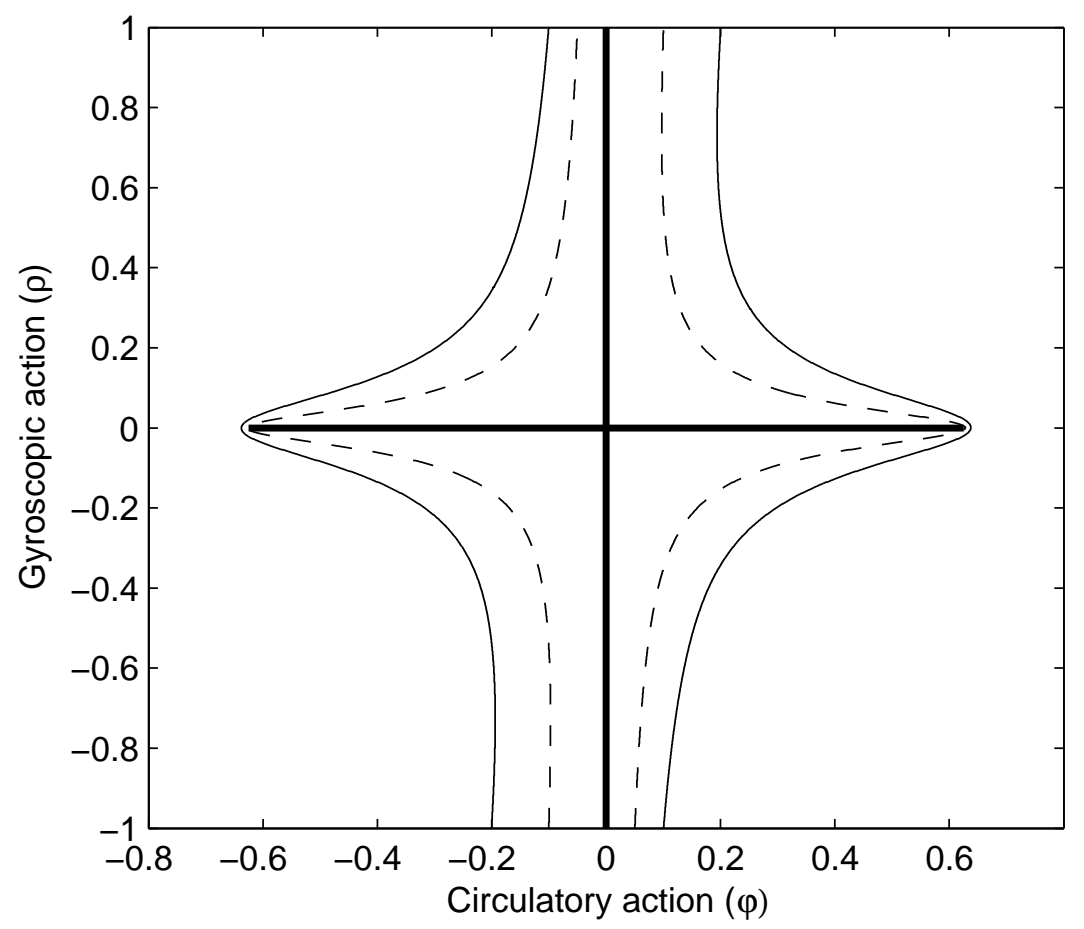

Figure 2: Stability frontiers for $\alpha=1.5$ and iso-distributed damping $(\alpha \beta=1)$, bold line: no damping, dashed line: $\xi_{t}=5 \%$, solid line: $\xi_{t}=10 \%$
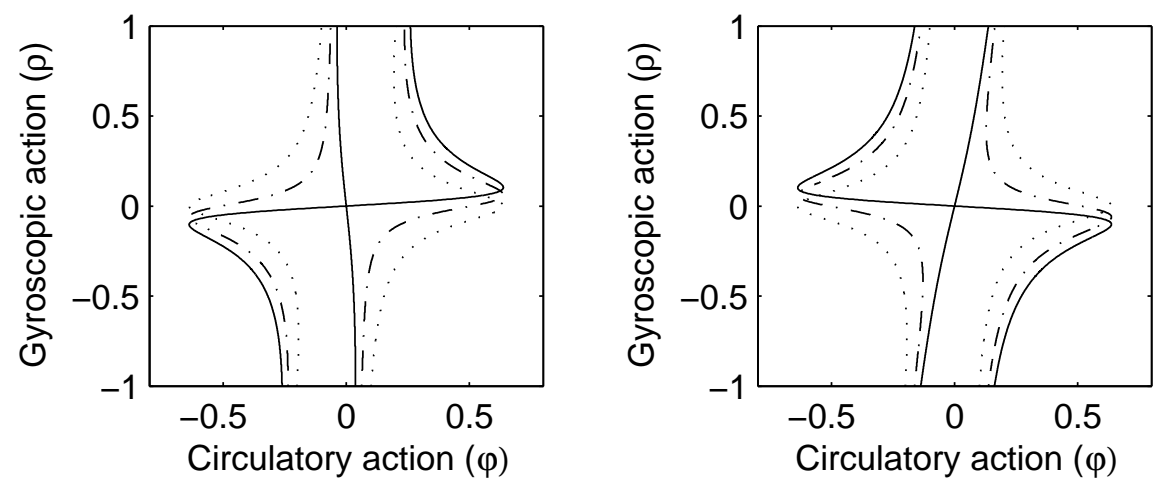

(a) Dot line: $\alpha \beta=1$, dot-dashed lined: $\alpha \beta=$ (b) Dot line: $\alpha \beta=1$, dot-dashed lined: $\alpha \beta=$ 4, solid line: $\alpha \beta \rightarrow \infty$

0.25 , solid line: $\alpha \beta=0$

Figure 3: Stability frontiers for $\alpha=1.5$, and $\xi_{t}=10 \%$ and various damping distributions 


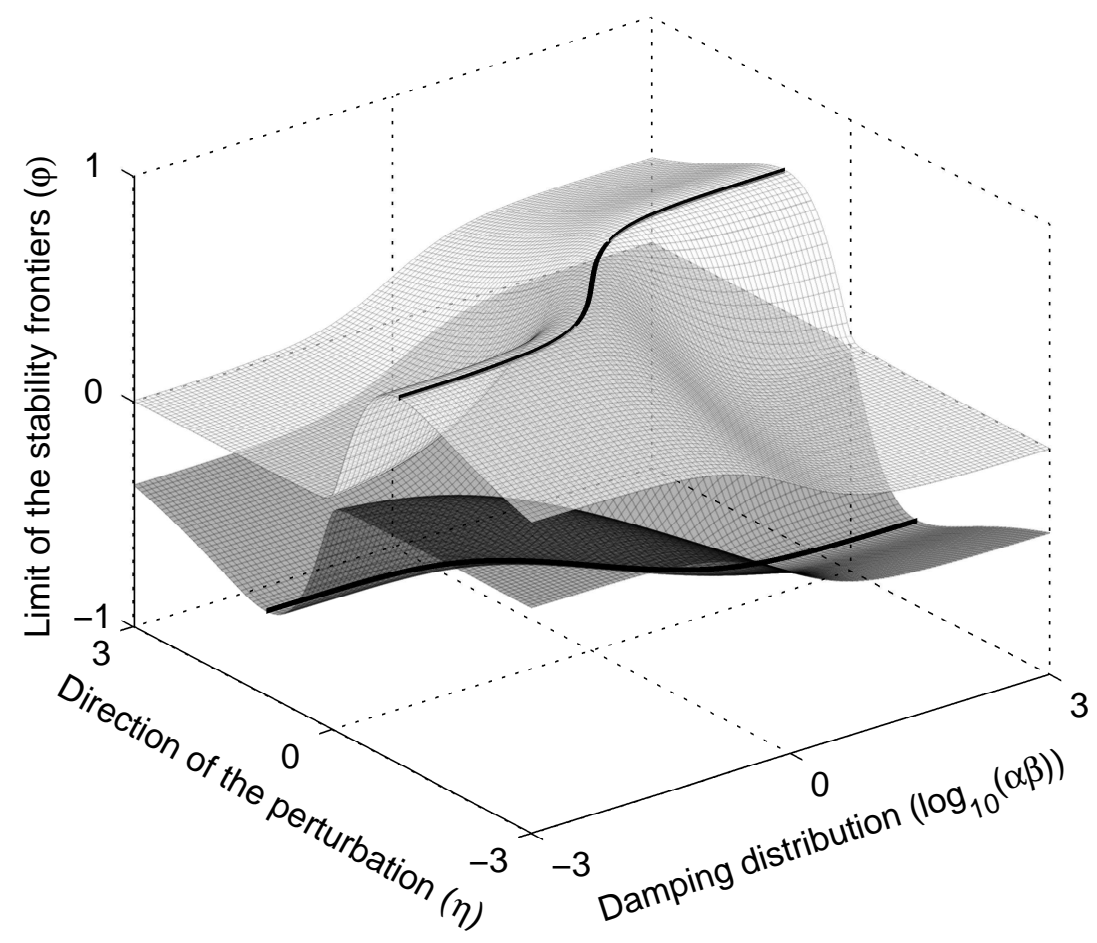

Figure 4: Limit of the stability frontiers for a vanishingly damped purely circulatory system versus the damping distribution and the gyroscopic contribution for $\alpha=1.5$, bold lines indicate the extrema with regard to $\eta$

the domain of marginal stability of the undamped system. Furthermore, looking for maxima of $|\varphi|$ for given $\alpha$ and $\beta$, one obtains

$$
\left|\varphi_{\max }\right|=\left(\frac{\alpha^{2}-1}{2}\right), \text { with } \varphi_{\max } \eta=\left(\frac{1-\alpha^{2}}{2}\right)\left(\frac{1-\alpha \beta}{1+\alpha \beta}\right) .
$$

This result indicates that the limit of the stability frontier when the perturbation vanishes is at most the limit of marginal stability of the unperturbed system (coalescence point), in accordance with [9]. This also reveals that the smaller the coefficients of the damping matrix are, the more important is to know the structure of this matrix in order to correctly predict the stability nature of the equilibrium, which constitutes the destabilization paradox. This makes the understanding of poorly damped systems a difficult matter.

As illustrated on Fig. 4, the locus of the extrema of $|\varphi|$ as $\xi_{t}$ vanishes correspond to two distinct curves described by the second part of Eq. 30. Indeed, a maximum of $|\varphi|$ exists for both positive and negative values. These curves cross each other at $\eta=0$ and $\alpha \beta=1$. At this point both limits reach their maximum and therefore the overall stability region is the widest.

A maximum width of the stability domain is also observed close to the purely circulatory system with an iso-distribution of damping for a non-vanishing damping on Fig. 3. As a matter of fact, the width $\Delta \varphi$ of the stability domain can be calculated from Eq. 28 and is a $C^{1}$ even function of $\rho$, which implicates that an extremum of $\Delta \varphi$ always exists at $\rho=0$. The authors have reported the optimal damping distribution 


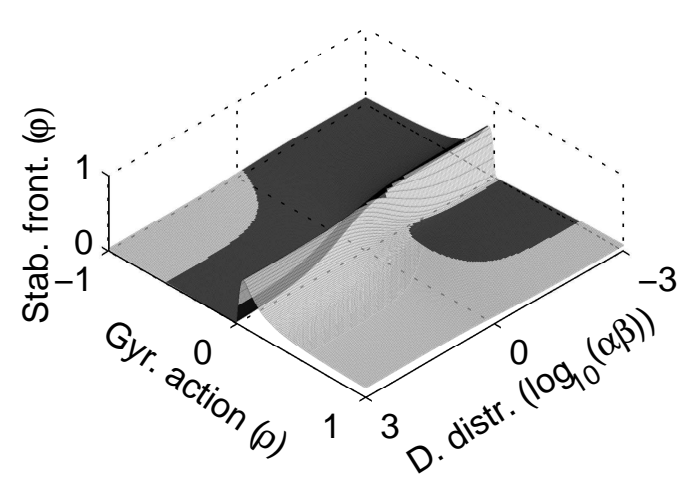

(a)

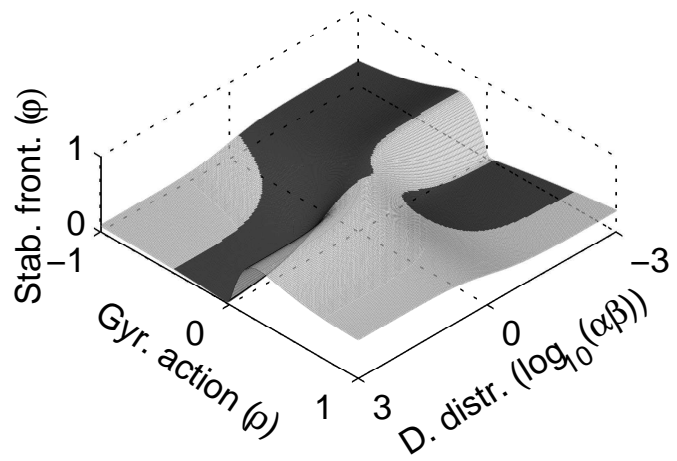

(b)

Figure 5: Stability frontiers for $\varphi>0$ and nature of the Hopf bifurcation versus the damping distribution and the gyroscopic factor for $\alpha=1.5, \xi_{t}=5 \%$ (a) and $\xi_{t}=20 \%$ (b) (dark: subcritical bifurcation, light: supercritical bifurcation)

which maximize $\Delta \varphi$ for $\rho=0$ in [13],

$$
(\alpha \beta)_{o p t}=\frac{\sqrt{16\left(\alpha^{4}-\alpha^{2}+1\right) \xi_{t}^{4}+4\left(\alpha^{2}+1\right)\left(\alpha^{2}-1\right)^{2} \xi_{t}^{2}+\left(\alpha^{2}-1\right)^{4}}-4\left(\alpha^{2}-1\right) \xi_{t}^{2}}{4 \xi_{t}^{2}+\left(\alpha^{2}-1\right)^{2}}
$$

which actually tends to $(\alpha \beta)_{\text {opt }}=1$ as $\xi_{t}$ vanishes. Therefore, the iso-distribution of damping can be considered as a "practical" criterion of optimization of the stability domain for quasi-purely circulatory systems. It can be observed on Fig. 2 that the local extreme values of the circulatory action on the frontier evolve very slowly in the vicinity of purely circulatory systems as the damping amount increases. This emphasizes the important role of the damping structure relatively to the damping amount.

In order to complete this overview of the stability domain, the nature of the Hopf bifurcation has been investigated from Eq. 1 and reported on Fig. 5 for two different amounts of damping. It can be observed on this figure that the damping distribution strongly affects the nature of the bifurcation. Contrariwise, the amount of damping has a weak influence. Once again, a dominant role of the damping structure is suggested by this result.

\section{Non-linear analysis and limit cycles}

Although the stability analysis is a powerful tool in order to highlight phenomenological roots of selfgenerated vibrations, the prediction of their properties definitely requires taking into account the nonlinearities of the system. To this purpose, a method for determining the limit cycles of autonomous nonlinear systems is introduced in the first part. Then, a stability analysis of these periodic solutions is proposed. This last step aims at predicting if the system is likely to converge to one of these solutions or if a more complex behaviour is to be expected. Finally, the limit cycles of the example model are investigated.

\subsection{Determination of the limit cycles}

A method is proposed in this section to identify the limit cycle arising from the Hopf bifurcation. This method is based on an extension of the harmonic balance method. It includes dynamic constraints in 
order to avoid convergence of the estimated solution to the equilibrium. It shares some similarities with the Constrained Harmonic Balance Method (CHBM) method proposed by Coudeyras et al. [15].

Assume a system described by

$$
\ddot{\mathbf{Y}}=\mathbf{h}(\mathbf{Y}, \dot{\mathbf{Y}}, \mathbf{p}) \text {, }
$$

where $\mathbf{h}$ is a real function globally continuous with regard to both the real vectors $\mathbf{Y}$ and $\dot{\mathbf{Y}}$, and a continuous and piecewise $C^{1}$ periodic solution exists. This solution can be expressed as a Fourier series,

$$
\mathbf{Y}(t)=\mathbf{A}_{\mathbf{0}}(\mathbf{p})+\sum_{n \geqslant 1}\left(\mathbf{A}_{\mathbf{n}}(\mathbf{p}) \cos (n \omega(\mathbf{p}) t)+\mathbf{B}_{\mathbf{n}}(\mathbf{p}) \sin (n \omega(\mathbf{p}) t)\right),
$$

where $\mathbf{A}_{n}(\mathbf{p})$ and $\mathbf{B}_{n}(\mathbf{p})$ are constant vectors and $\omega(\mathbf{p})$ is the angular frequency of the solution. In the previous expression, $\omega(\mathbf{p})$ is unknown since it is likely to differ from the angular frequency of the unstable mode obtained by the eigenvalues analysis of the linearized system.

The method consists in changing the time integration problem into an optimization problem. Consider the following functional,

$$
\Psi\left(\left[\mathbf{A}_{n}\right],\left[\mathbf{B}_{n}\right], \omega\right)=\int_{t_{0}}^{t_{0}+2 \pi / \omega}\|\ddot{\mathbf{Y}}(t)-\mathbf{h}(\mathbf{Y}(t), \dot{\mathbf{Y}}(t), \mathbf{p})\|^{2} \mathrm{~d} t .
$$

A solution of Eq. 32 is a global minimum of $\Psi$. In order to numerically identify it, the Fourier series is truncated at a high enough order. By doing so, the functional is changed into a function of a finite number of variables. Finally, convergence to some irrelevant local minima (as equilibrium that is an exact solution of the nonlinear expression of Eq. 32) can be avoided if the following constraints are imposed:

$$
\begin{gathered}
\int_{t_{0}}^{t_{0}+2 \pi / \omega}(\ddot{\mathbf{Y}}(t)-\mathbf{h}(\mathbf{Y}(t), \dot{\mathbf{Y}}(t), \mathbf{p})) \mathrm{d} t=\mathbf{0}, \\
\int_{t_{0}}^{t_{0}+2 \pi / \omega(\mathbf{p})}\left|\ddot{\mathbf{Y}}_{i}(t)\right| \mathrm{d} t-\int_{t_{0}}^{t+2 \pi / \omega}\left|\mathbf{h}_{i}(\mathbf{Y}(t), \dot{\mathbf{Y}}(t), \mathbf{p})\right| \mathrm{d} t=0, \forall i, \\
\int_{t_{0}}^{t_{0}+2 \pi / \omega(\mathbf{p})} \frac{\left(\ddot{\mathbf{Y}}_{i}(t)+\mathbf{h}_{i}(\mathbf{Y}(t), \dot{\mathbf{Y}}(t), \mathbf{p})\right) \dot{\mathbf{Y}}_{i}(t)}{\sqrt{\dot{\mathbf{Y}}_{i}(t)^{2}+\ddot{\mathbf{Y}}_{i}(t)^{2}}} \mathrm{~d} \tau=0, \forall i,
\end{gathered}
$$

where the subscript $i$ indicates the $i$-th component of a vector. Theses proposed constraints correspond to a null mean error, to a mean respect of the fundamental principle of dynamics on each DOF and to a motion being neither dilating nor contracting in the phase plane of each DOF respectively. Other constraints can be used to improve the convergence, provided they correspond to some properties fulfilled by both the solution and its approximations.

By solving this problem, not only one finds an approximate form of the solution but also the angular frequency is identified. Moreover, this approach allows obtaining estimations faster than a direct time integration of Eq. 32, unstable limit cycles can be identified as well, and no post-processing is required. The method is particularly efficient with parametric studies of continuous limit cycle branches when one iteratively uses a result as an initial guess for a close new set of parameters. 


\subsection{Stability analysis of the limit cycles}

Assume a periodic solution $\mathbf{X}$ of Eq. 4 has been identified having the angular frequency $\omega=2 \pi / T$ with $T$ its period. The analysis of its stability aims at determining if this solution is attractive or not, i.e. if the system is likely to converge or not to this limit cycle. To this purpose, a vanishing perturbation $\varepsilon$ of $\mathbf{X}$ is considered and $\tilde{\mathbf{X}}=\mathbf{X}+\varepsilon$ denotes the perturbed solution. Then, one considers the following local approximation of Eq. 4 in the vicinity of $\mathbf{X}$

$$
\dot{\varepsilon} \approx \mathrm{D} \mathbf{f}(\mathbf{X}, \mathbf{p}) \varepsilon,
$$

which is a periodic non-autonomous but linear differential equation. Therefore, its solutions form a vector space. Consider a basis of solution $\left\{\mathbf{e}_{i}\right\}$. Because of the periodicity of Eq. 38, one can express the elements of this base as linear combinations of the same elements translated of one period in time,

$$
\mathbf{e}_{i}(t+T)=\sum_{j \geqslant 1} c_{i j} \mathbf{e}_{j}(t), \forall i, \forall t \text { or } \mathbf{E}(t+T)=\mathbf{M E}(t), \forall t \text { with } \mathbf{E}=\left[\mathbf{e}_{i}\right]
$$

The constant matrix $\mathbf{M}$ is the monodromy matrix. The basis $\left\{\mathbf{e}_{i}\right\}$ can be chosen so that

$$
\mathbf{E}\left(t_{0}\right)=\mathbf{I},
$$

where $\mathbf{I}$ is the identity matrix and $t_{0}$ is an arbitrary origin of time. It follows that

$$
\mathbf{M}=\mathbf{E}\left(t_{0}+T\right) .
$$

The eigenelements of $\mathbf{M}$ characterize the modes of perturbation of $\mathbf{X}$. An eigenvector $\mathbf{V}_{i}$ associated to the eigenvalue $\mu_{i}$ is such that $\mathbf{M} \mathbf{V}_{i}=\mu_{i} \mathbf{V}_{i}$, i.e. $\mathbf{V}_{i}(t+T)=\mu_{i} \mathbf{V}_{i}(t)$. Therefore, according to the Floquet theory

$$
\mathbf{V}_{i}(t)=\Theta_{i}(t) \mathrm{e}^{i \frac{\arg \left(\mu_{i}\right)}{T} t} e^{\frac{\log \left(\left|\mu_{i}\right|\right)}{T} t},
$$

where $\Theta_{i}(t)$ is a $T$-periodic function. It follows that the studied limit cycle is unstable if at least one eigenvalue of $\mathbf{M}$ has a modulus strictly bigger than 1. Because Eq.4 is autonomous, any translation in time of $\mathbf{X}$ produces a new solution. The difference between this new solution and $\mathbf{X}$ corresponds to a periodic perturbation. Therefore, 1 is always an eigenvalue of $\mathbf{M}$.

Assume one of the other eigenvalue crosses the unit circle and the remaining ones remain inside. Then, it can be shown from the center manifold theory that the crossing through 1 corresponds to the junction of a stable limit cycle with an unstable limit cycle which both disappear. A crossing through -1 corresponds to the destabilization of the limit cycle and the appearance of period-doubling bifurcation. Finally, a crossing by a pair of non-real conjugate eigenvalue corresponds to the junction of a stable limit cycle with a stable biperiodic trajectory (Neimark-Sacker bifurcation).

It can be observed that this stability analysis of the limit cycle is similar to the stability analysis of equilibriums introduced in the previous section and can even be considered as its generalization. Indeed, equilibrium can be considered as a periodic solution with any value for $T$ and the same approach can be used to define the stability. This consideration leads to

$$
\mathbf{M}=\mathrm{e}^{\mathrm{D} \mathbf{f}\left(\mathbf{X}_{e}\left(\mathbf{p}_{0}\right), \mathbf{p}_{0}\right) T} .
$$

A translation in time of equilibrium induces no difference so there is no reason for $\mathbf{M}$ to admit 1 as an eigenvalue, except on the stability frontier. According to the center manifold theory, the period of the destabilizing mode and the period of the limit cycle arising from the Hopf bifurcation converge to each other at the bifurcation point. Therefore, if $T$ is chosen so that it is equal to this common period at the bifurcation point, the exponential of the eigenvalues of $\mathrm{D} \mathbf{f}\left(\mathbf{X}_{\mathbf{e}}\left(\mathbf{p}_{\mathbf{0}}\right), \mathbf{p}_{\mathbf{0}}\right)$ time $T$ can be expected to continuously connect with the eigenvalues of the monodromy matrix of the limit cycle at the bifurcation point. 

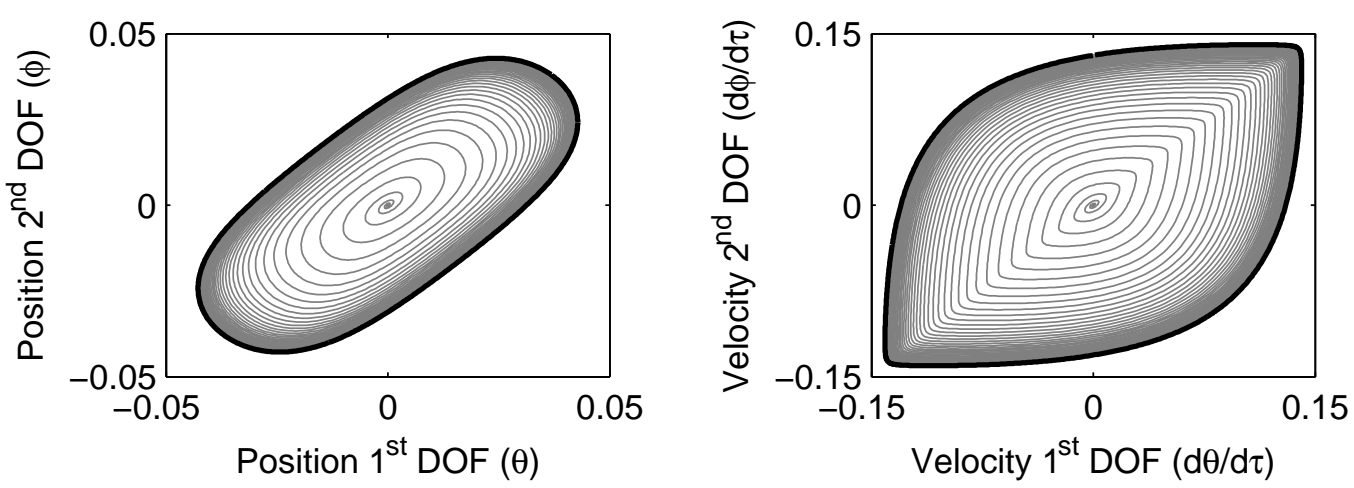

Figure 6: Comparison between time integration and limit cycle estimation (gray: time integration, black: estimated limit cycle) of Eq. 1 with $\alpha=1.5, \alpha \beta=1, \xi_{t}=20 \%, \varphi=1, \rho=0$ and $\sigma=10^{4}$, (a) trajectory (b) velocity phase

\subsection{Parametric investigation of the limit cycles of the squealing clutch}

The proposed method is applied at order 9 (truncation order of the Fourier series) that allows a high enough precision over the domain of investigation, and the results are compared to direct time integration on Fig. 6 for $\xi_{t}=20 \%, \alpha \beta=1, \rho=0$ and $\varphi=1$. It is observed on the whole results that the qualitative results are rather independent from the damping amount. $\xi_{t}=20 \%$ is a high value of damping. However, high values are observed to allow obtaining smoother evolutions of the limit cycles. Therefore, this example is more appropriate for preliminary observations. More realistic damping amounts are investigated later.

Both the trajectory of the identified limit cycle in the state space and the estimated angular frequency are in very good accordance with the result from time integration. It can be noted in this example that the angular frequency of the limit cycle is $\omega=3.85{\mathrm{rad} . \mathrm{s}^{-1}}^{-1}$ (see Fig. 5(b)) which greatly differs from the angular frequency of the unstable linear mode $\omega_{0}=1.31$ rad.s ${ }^{-1}$ obtained by considering the stability analysis of the linearized system.

Following Eq. 38, a local approximation of Eq. 1 in the vicinity $\varepsilon=\left[\begin{array}{ll}\varepsilon_{1} & \varepsilon_{2}\end{array}\right]^{T}$ of a limit cycle $\mathbf{X}=\left[\begin{array}{ll}\theta & \phi\end{array}\right]^{T}$ can be expressed as

$$
\left[\begin{array}{c}
\ddot{\varepsilon}_{1} \\
\ddot{\varepsilon}_{2}
\end{array}\right]+\left[\begin{array}{cc}
2 \xi & \rho \\
-\rho & 2 \alpha \beta \xi
\end{array}\right]\left[\begin{array}{c}
\dot{\varepsilon}_{1} \\
\dot{\varepsilon}_{2}
\end{array}\right]+\left[\begin{array}{cc}
1 & \varphi \\
-\varphi & \alpha^{2}
\end{array}\right]\left[\begin{array}{l}
\varepsilon_{1} \\
\varepsilon_{2}
\end{array}\right]+3 \sigma\left[\begin{array}{l}
\theta^{2} \varepsilon_{1} \\
\phi^{2} \varepsilon_{2}
\end{array}\right]=\left[\begin{array}{l}
0 \\
0
\end{array}\right] .
$$

The eigenvalues of the monodromy matrix obtained for the example by integrating Eq. 44 (see Fig. 7(c) and 7(d)) are inside the unit circle, which confirms the stable nature of the identified limit cycle.

The limit cycles can be repeatedly estimated in the same manner in order to perform a parametric investigation. Such a parametric investigation is reported on Fig. 7 for a variation of the circulatory action and on Fig. 8 for a variation of the gyroscopic action for the same values of the other parameters as in the previous example.

The mean inclination indicated on these figures is the mean inclination of the disc over one period of the identified limit cycles as expressed in Eq. 45. This expression correspond to the instantaneous global inclination of the disc (combination of the two components) averaged over one period of the periodic motion. The associated stability nature corresponds either to stability if no eigenvalues of the monodromy matrix has a modulus bigger than 1 , instability with a dominant real eigenvalue if the biggest eigenvalues in modulus is real or instability with a pair of dominant conjugate complex eigenvalue if the 

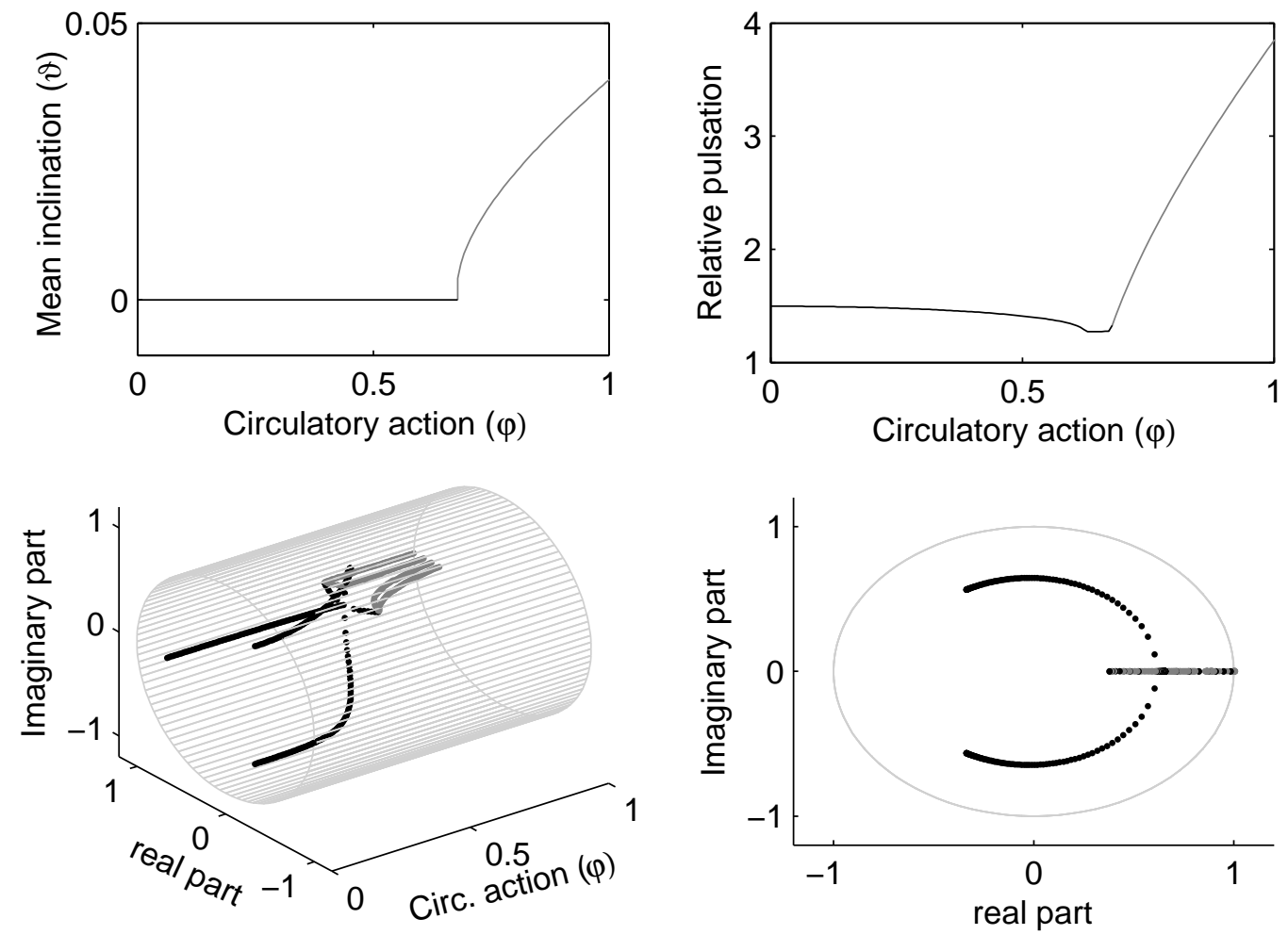

Figure 7: Equilibrium in its stability domain (black) connecting to the arising limit cycle (gray) versus the circulatory action, for Eq. 1 with $\alpha=1.5, \alpha \beta=1, \xi_{t}=20 \%, \rho=0$ and $\sigma=10^{4}$, (a) mean inclination, (b) relative frequency (least stable mode and limit cycle respectively), (c) and (d) eigenvalues of the monodromy matrix and unit cylinder 

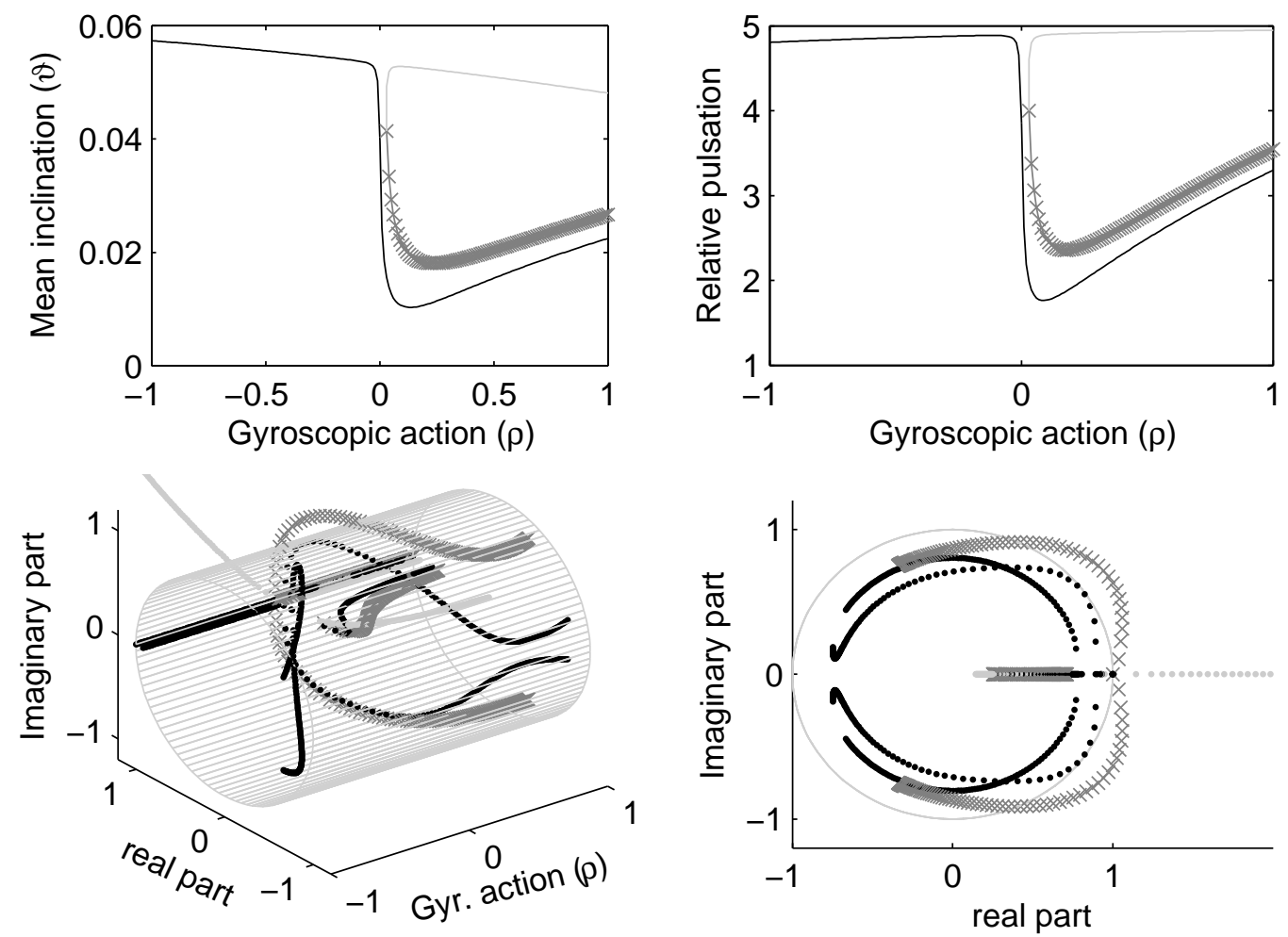

Figure 8: Limit cycles versus the gyroscopic action (gray, gray with crosses and black colors intend to help distinguishing the branches of limit cycle), for Eq. 1 with $\alpha=1.5, \alpha \beta=1, \xi_{t}=20 \%, \varphi=1$ and $\sigma=10^{4}$, (a) mean inclination, (b) relative frequency, (c) and (d) eigenvalues of the monodromy matrix and unit cylinder 
biggest eigenvalues in modulus is complex.

$$
\vartheta=\frac{\omega}{2 \pi} \int_{\tau=\tau_{0}}^{\tau=\tau_{0}+\frac{2 \pi}{\omega}} \sqrt{\theta^{2}+\phi^{2}} \mathrm{~d} \tau .
$$

Regarding the evolution of the limit cycle versus the circulatory action for the purely circulatory system, see Fig. 7, one can observe a unique limit cycle arising at the Hopf bifurcation with growing amplitude and frequency. The eigenvalues of the monodromy matrix of the limit cycle continuously connect with those of the equilibrium at the bifurcation point as expected. Then, one eigenvalue is equal to 1 and the other ones remain inside the unit circle, which indicates a stable limit cycle on the studied domain.

From the end of this domain $(\varphi=1)$ and by varying the gyroscopic action in the vicinity of 0 , see Fig. 8 , one can observe an abrupt evolution of both the amplitude and the frequency, and the appearance of a new limit cycle branch for $\rho>0.03$. The eigenvalues of the monodromy matrix indicate that the first branch remains always stable whereas the new one shows instability by real eigenvalues for the highest amplitude part and instability by complex eigenvalues then stability for the lowest amplitude part.

These figures illustrate a strong influence of the gyroscopic action on the existence of limit cycle branches and their evolution, especially in the vicinity of the purely circulatory system for an iso-distribution of damping. Indeed, a low gyroscopic action drastically modifies the limit cycle. Finally, it is important to observe on Fig. 8 that the frequency can either increase or decrease as the amplitude grows. Thus the frequency and the amplitude of the limit cycles are rather independent and show no simple relation.

Fig. 9-26 allow extending these observations to various values of damping amount, damping distribution, gyroscopic and circulatory actions (the equilibrium is not reported anymore on these figures). These figures show sections of the parameters space in the same manner as on Fig. 7-8 for $\xi_{t}=5 \%, \xi_{t}=10 \%$ and $\xi_{t}=20 \%$. It can be firstly checked that the subcritical or supercritical nature of the Hopf bifurcation observed on all these figures is in perfect accordance with Fig. 5.

The limit cycle branches and their stability domains tend to be more separated in the parameters space as the damping amount increases. However, no important qualitative effect is observed on their shape and relative positions. The most important effect of the damping amount is quantitative: the more damping the lower amplitude and frequency of the limit cycles. The influence of the circulatory action has also a general tendency, in spite of local discrepancies on unstable limit cycles: the more circulatory action, the larger amplitude and frequency.

The fast evolution of the limit cycle with regard to the gyroscopic action in the vicinity of the purely circulatory system with an iso-distributed damping which was previously mentioned can be generalized. Indeed, it can be observed for any damping amount and an iso-distribution of damping that when the purely circulatory system shows an unstable equilibrium, a change in the gyroscopic action leads to a fast evolution of the limit cycle, see Fig. 19, 22 and 25. For negative values of the gyroscopic action, a unique limit cycle with a large amplitude and frequency is observed. This limit cycle evolves slowly for large (negative) values of the gyroscopic action. For positive values of the gyroscopic action, the amplitude rapidly decreases, reaches a minimum then increases slowly. For a large enough gyroscopic action, a new limit cycle branch can be observed with the same qualitative behaviour as previously discussed. Fig. 22 suggests that the highest amplitude part and the lowest amplitude part of this new branch are likely to connect to form a closed branch for a large enough gyroscopic action. The highest amplitude part of this new branch is unstable by a dominant real eigenvalue of the monodromy matrix whereas the lowest amplitude part is firstly unstable by complex eigenvalues then becomes stable and its amplitude and frequency are close to those of the first branch over a range of the gyroscopic action.

For a lower circulatory action, i.e. when the purely circulatory system with an iso-distributed damping has a stable equilibrium, the first branch gets separated into two parts which surround the stable equilibrium. In this situation a negative gyroscopic action leads to a subcritical bifurcation with an important 

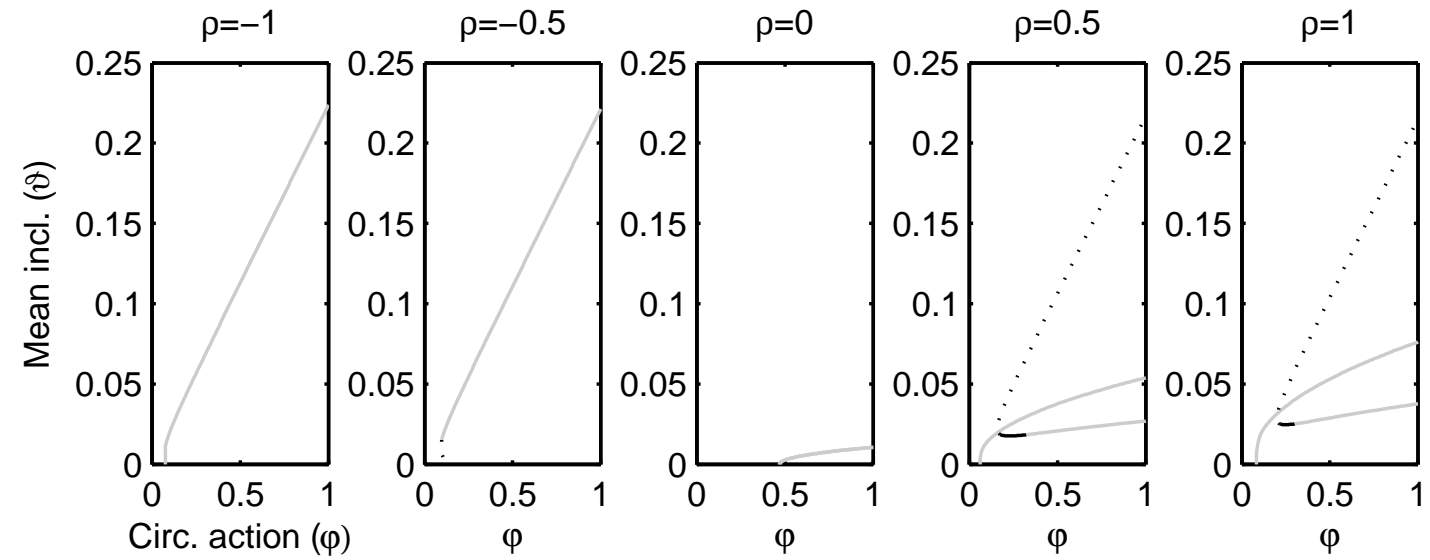

(a)
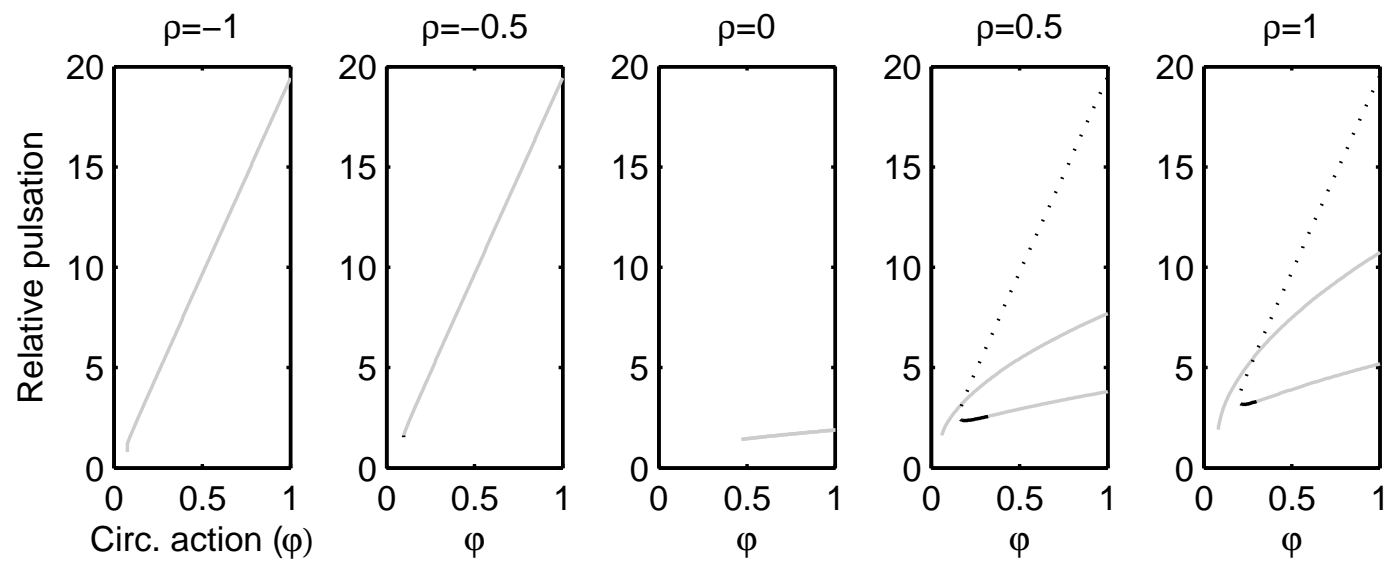

(b)

Figure 9: Limit cycles versus the circulatory action for various values of the gyroscopic action, for Eq. 1 with $\alpha=1.5, \alpha \beta=0.2, \xi_{t}=5 \%$ and $\sigma=10^{4}$, (a) mean inclination, (b) Relative frequency (gray: stable limit cycle, dot black: dominant real unstable eigenvalue, solid black: dominant pair of conjugate complex unstable eigenvalues) 

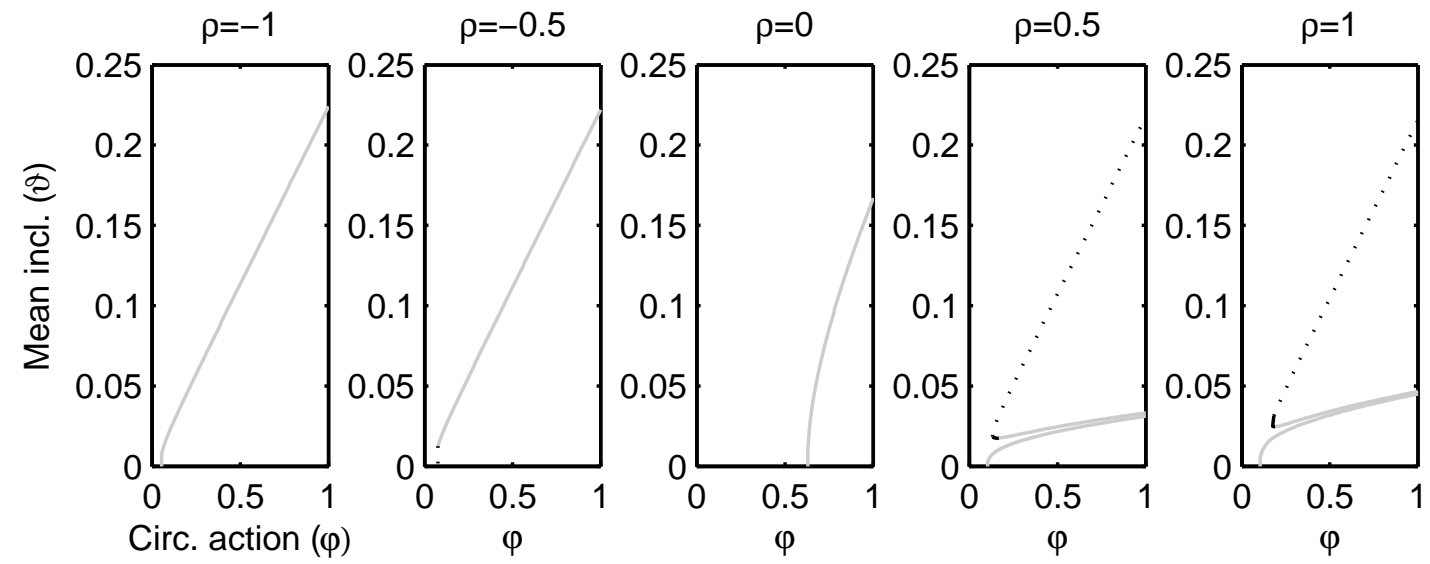

(a)
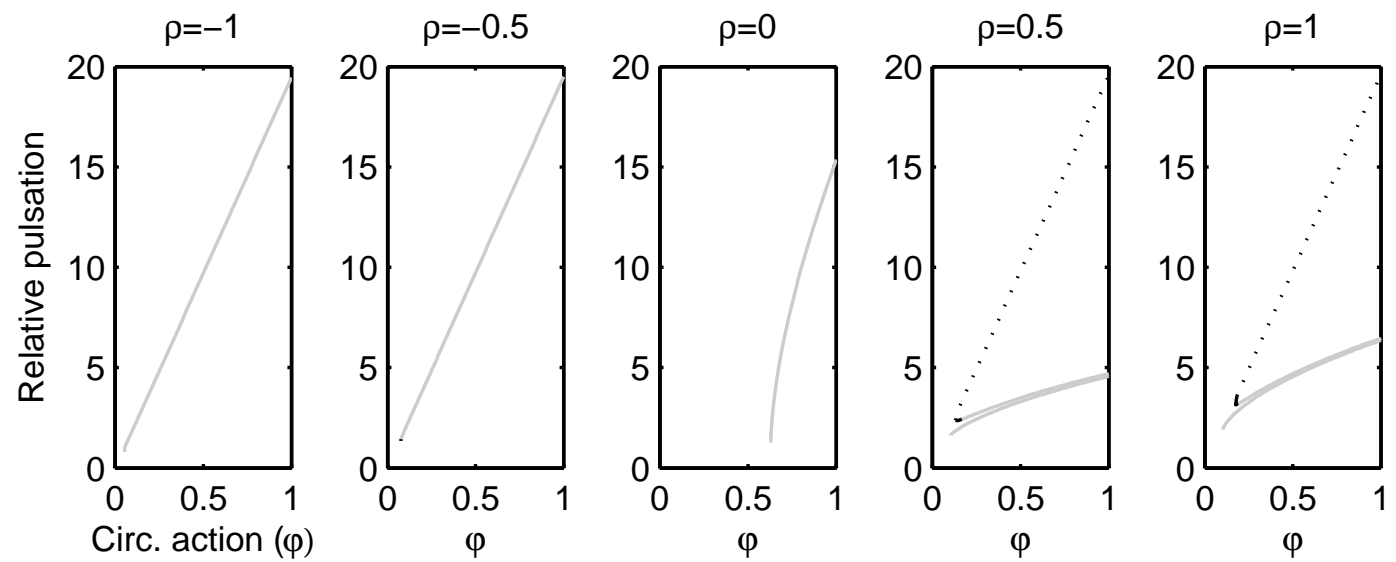

(b)

Figure 10: Limit cycles versus the circulatory action for various values of the gyroscopic action, for Eq. 1 with $\alpha=1.5, \alpha \beta=1, \xi_{t}=5 \%$ and $\sigma=10^{4}$, (a) mean inclination, (b) Relative frequency (gray: stable limit cycle, dot black: dominant real unstable eigenvalue, solid black: dominant pair of conjugate complex unstable eigenvalues) 

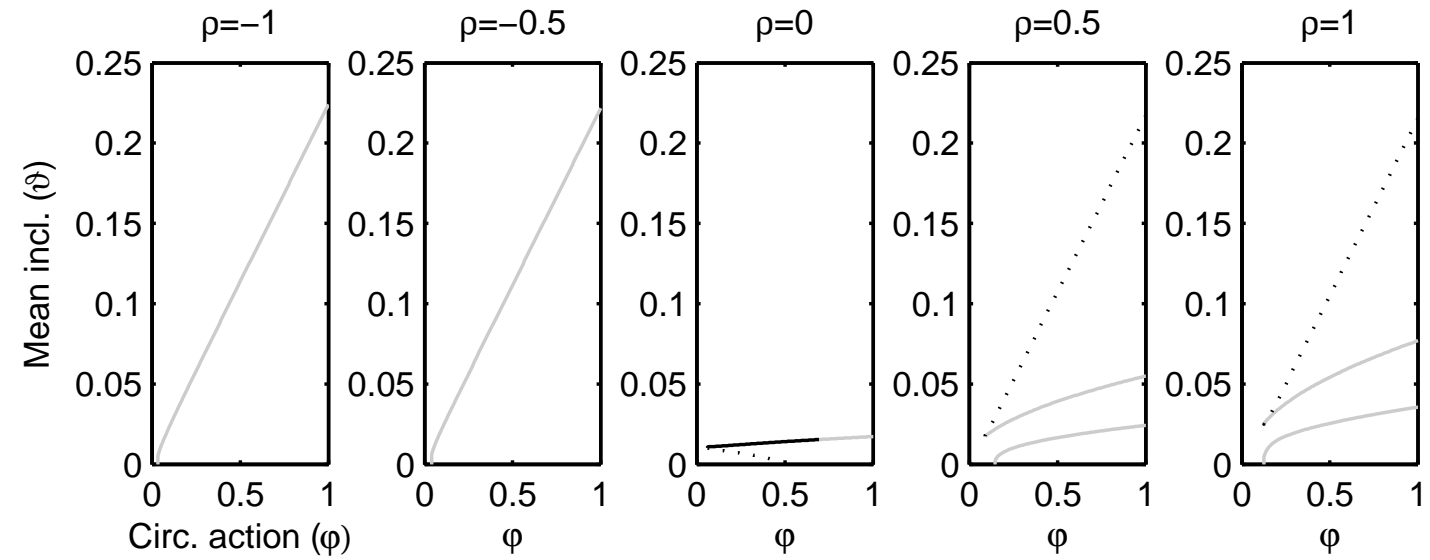

(a)
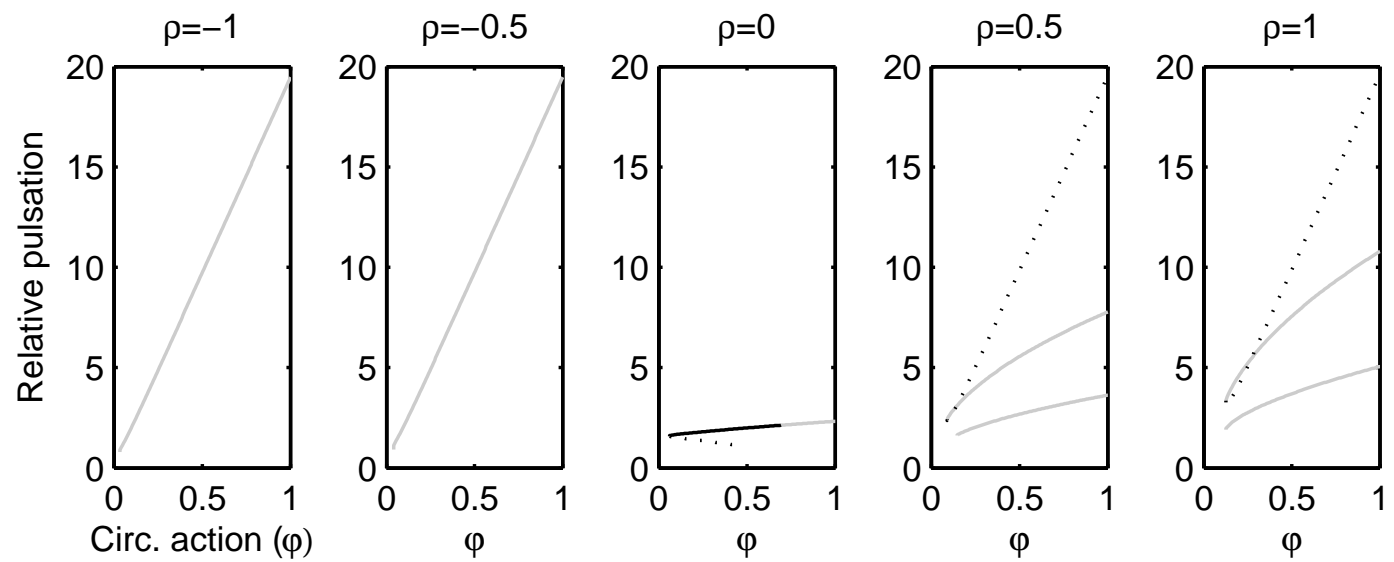

(b)

Figure 11: Limit cycles versus the circulatory action for various values of the gyroscopic action, for Eq. 1 with $\alpha=1.5, \alpha \beta=5, \xi_{t}=5 \%$ and $\sigma=10^{4}$, (a) mean inclination, (b) Relative frequency (gray: stable limit cycle, dot black: dominant real unstable eigenvalue, solid black: dominant pair of conjugate complex unstable eigenvalues) 

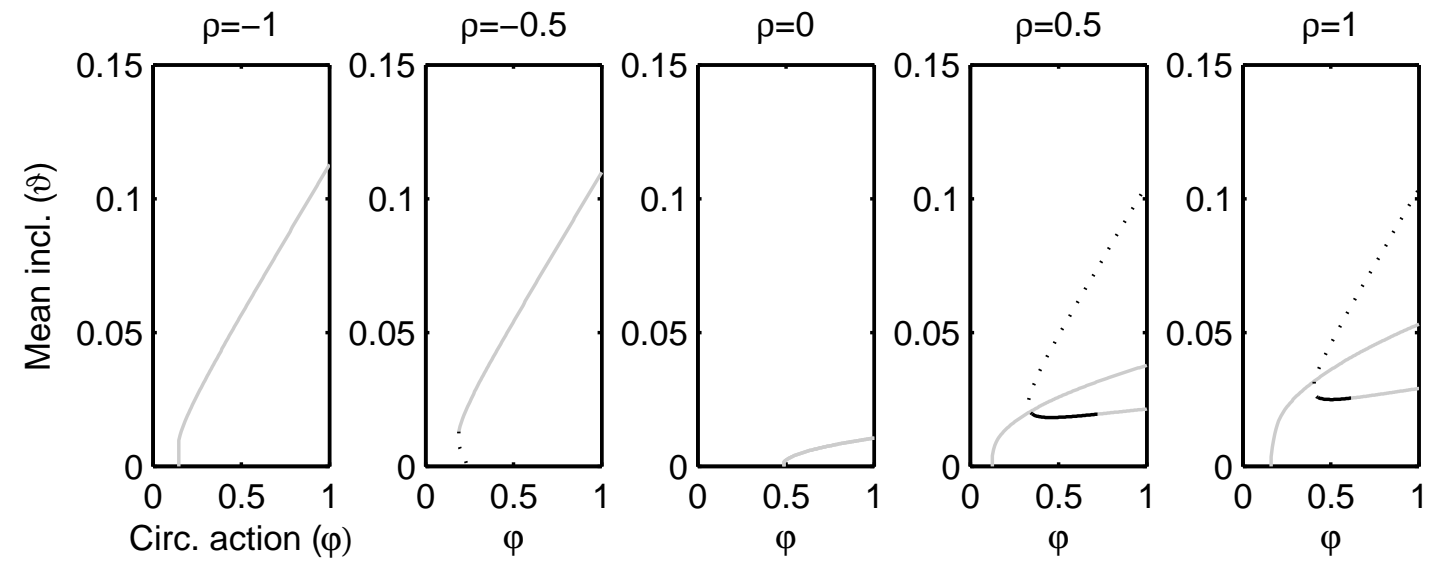

(a)
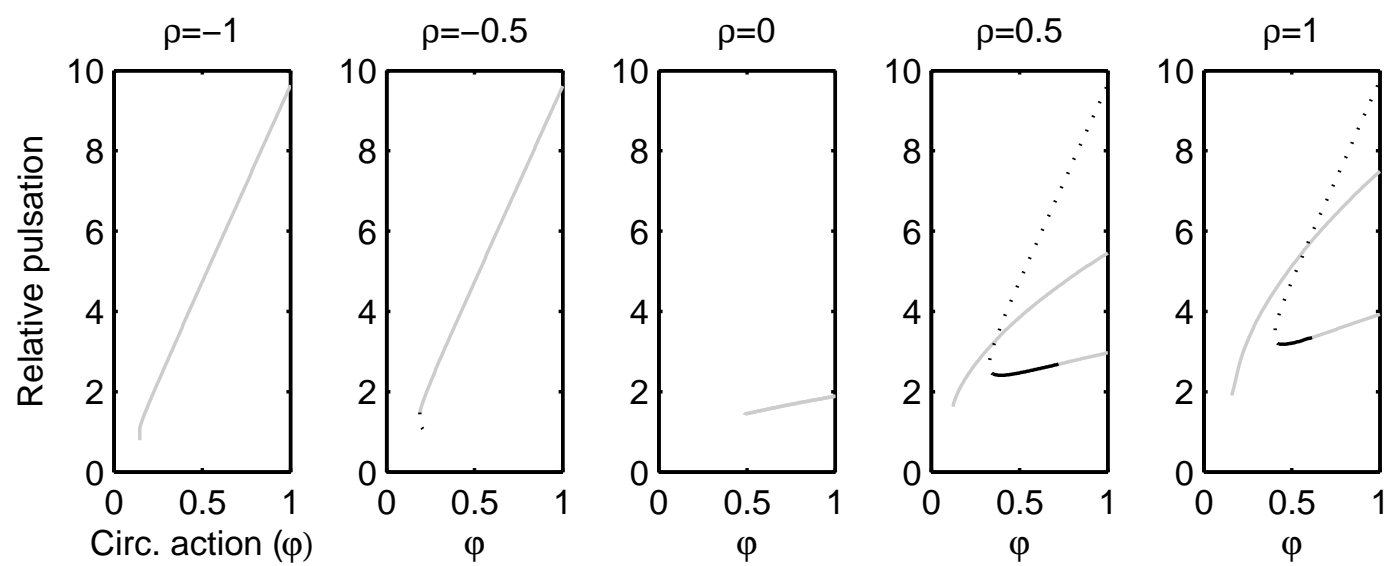

(b)

Figure 12: Limit cycles versus the circulatory action for various values of the gyroscopic action, for Eq. 1 with $\alpha=1.5, \alpha \beta=0.2, \xi_{t}=10 \%$ and $\sigma=10^{4}$, (a) mean inclination, (b) Relative frequency (gray: stable limit cycle, dot black: dominant real unstable eigenvalue, solid black: dominant pair of conjugate complex unstable eigenvalues) 

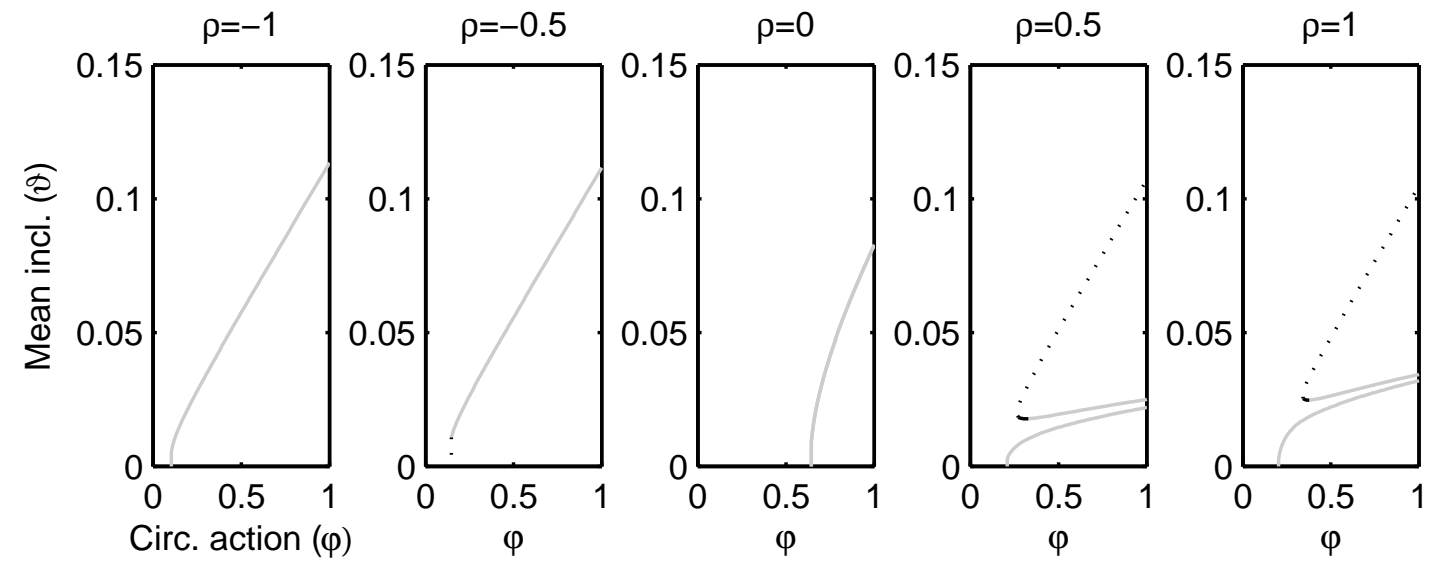

(a)
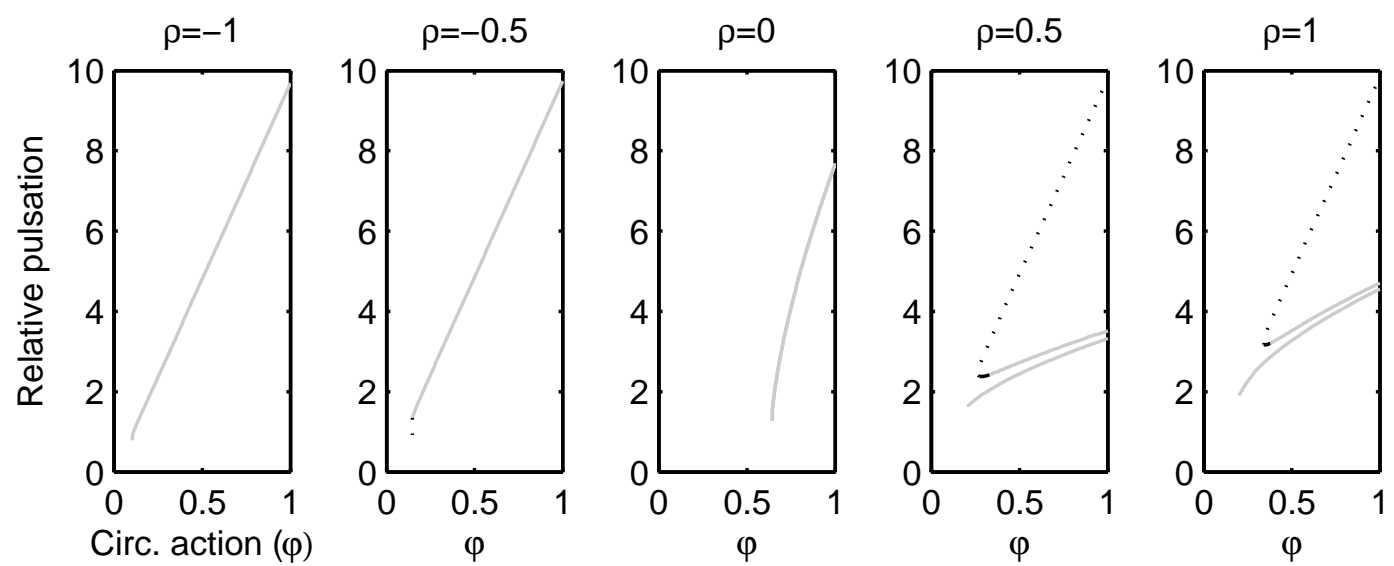

(b)

Figure 13: Limit cycles versus the circulatory action for various values of the gyroscopic action, for Eq. 1 with $\alpha=1.5, \alpha \beta=1, \xi_{t}=10 \%$ and $\sigma=10^{4}$, (a) mean inclination, (b) Relative frequency (gray: stable limit cycle, dot black: dominant real unstable eigenvalue, solid black: dominant pair of conjugate complex unstable eigenvalues) 

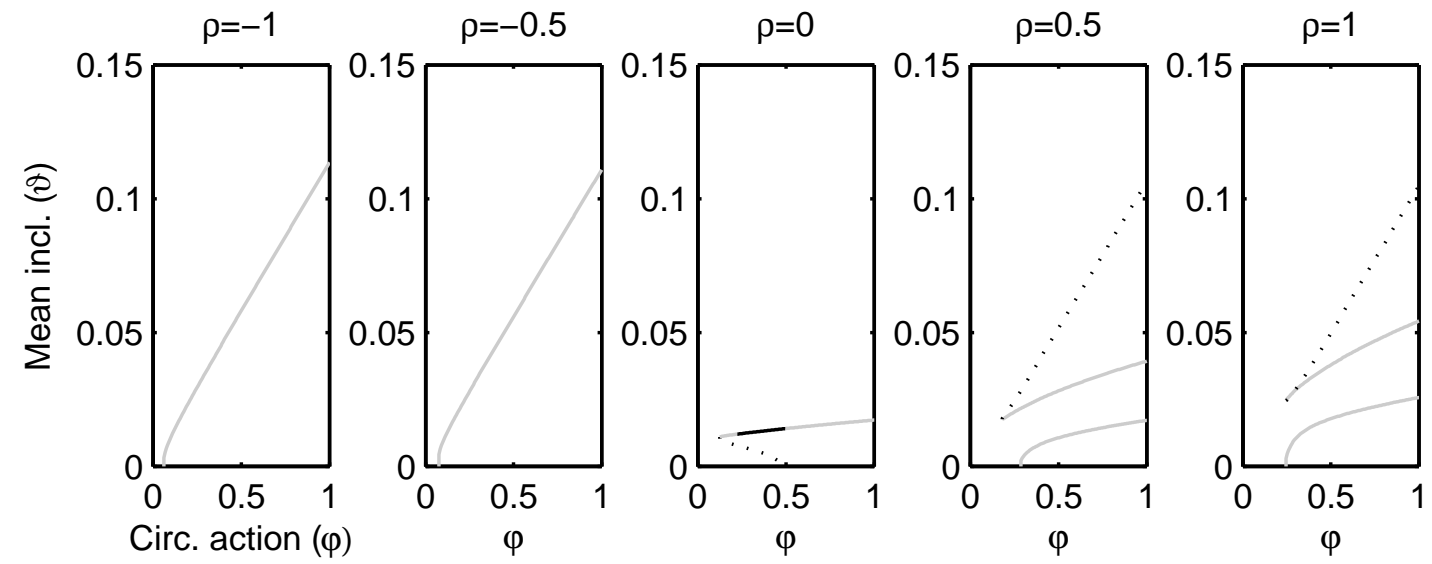

(a)
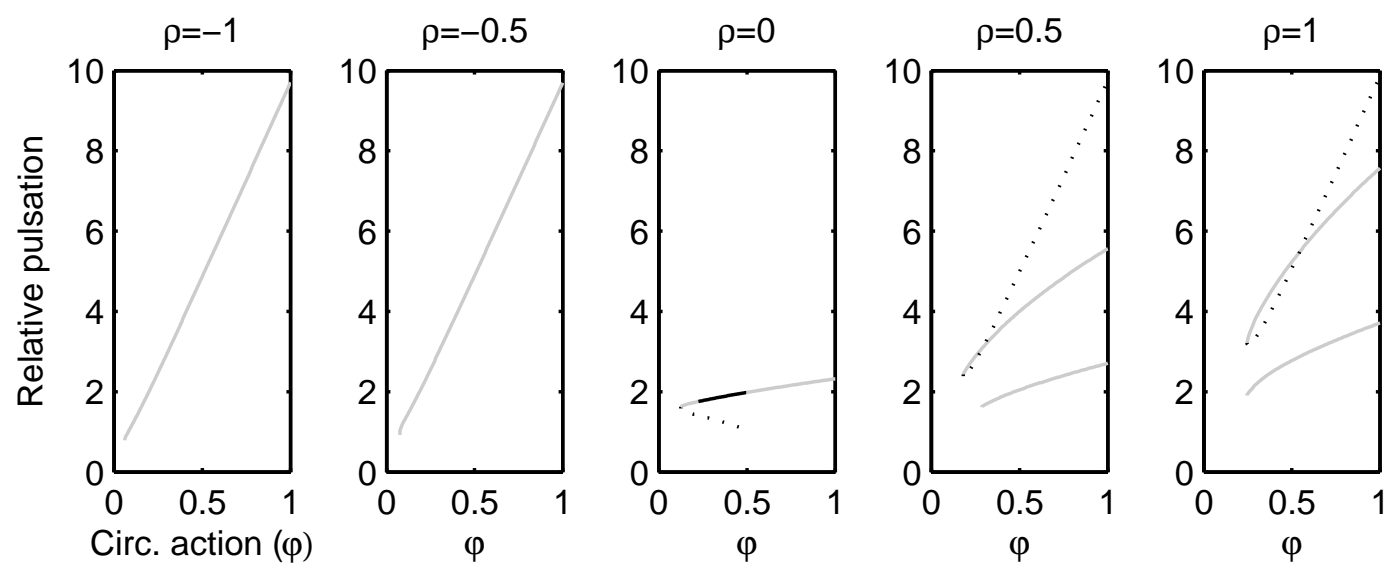

(b)

Figure 14: Limit cycles versus the circulatory action for various values of the gyroscopic action, for Eq. 1 with $\alpha=1.5, \alpha \beta=5, \xi_{t}=10 \%$ and $\sigma=10^{4}$, (a) mean inclination, (b) Relative frequency (gray: stable limit cycle, dot black: dominant real unstable eigenvalue, solid black: dominant pair of conjugate complex unstable eigenvalues) 


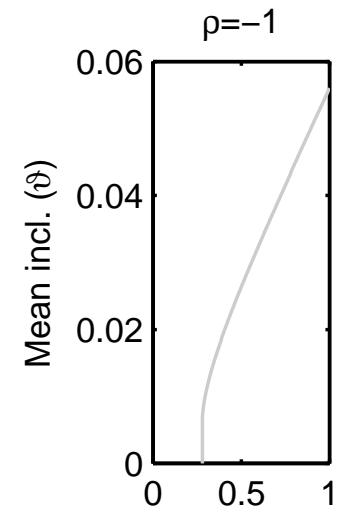

Circ. action $(\varphi)$
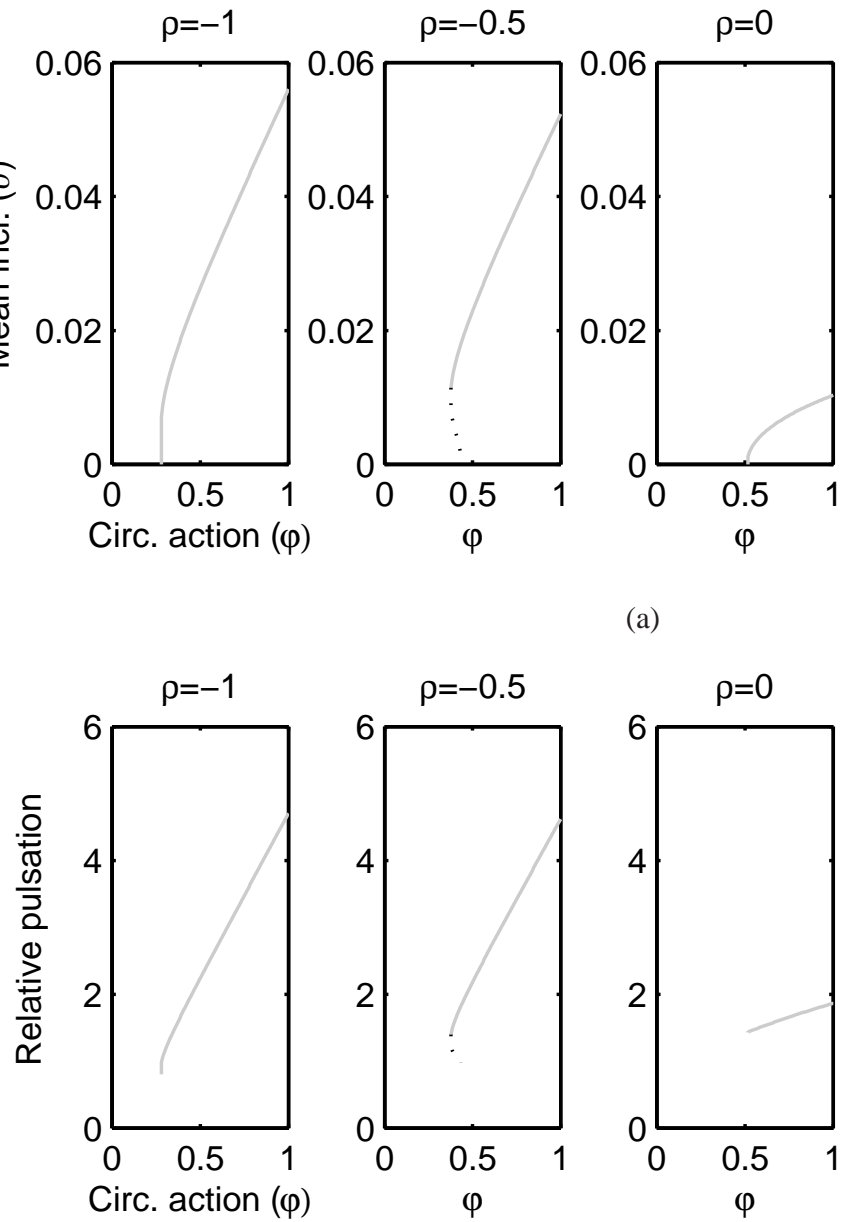

(a)
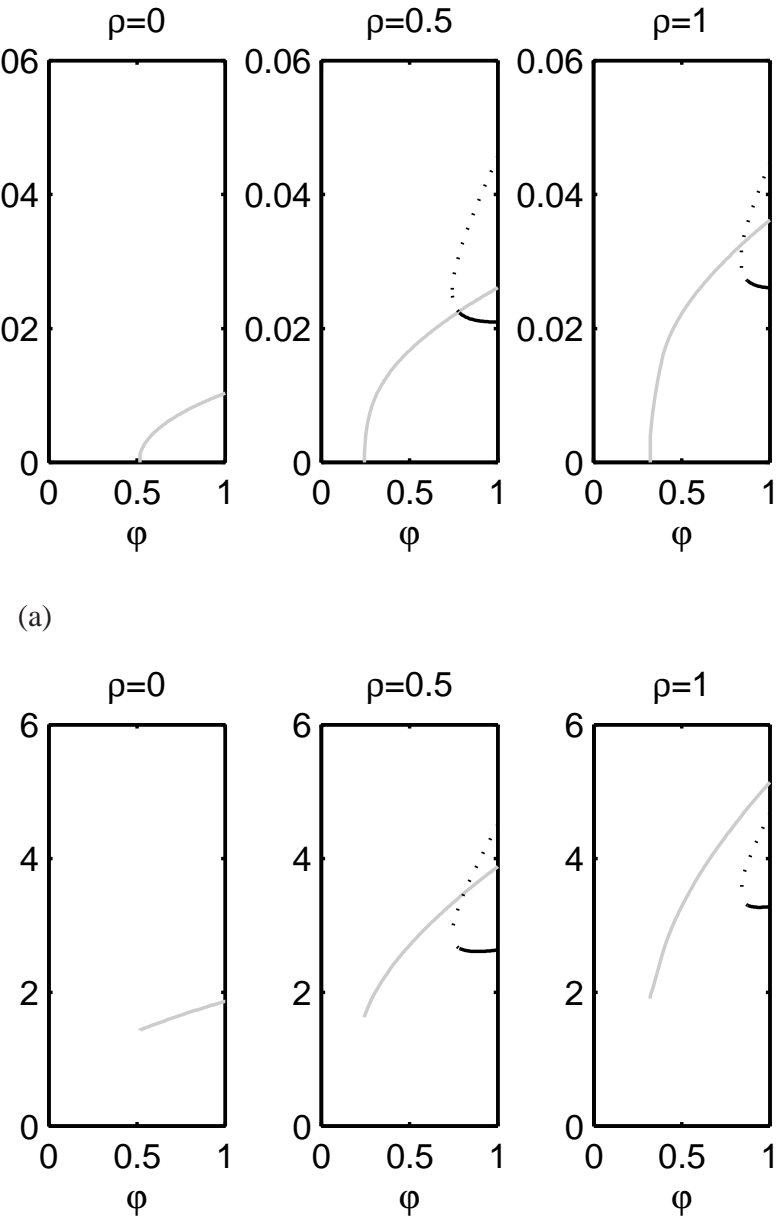

(b)

Figure 15: Limit cycles versus the circulatory action for various values of the gyroscopic action, for Eq. 1 with $\alpha=1.5, \alpha \beta=0.2, \xi_{t}=20 \%$ and $\sigma=10^{4}$, (a) mean inclination, (b) Relative frequency (gray: stable limit cycle, dot black: dominant real unstable eigenvalue, solid black: dominant pair of conjugate complex unstable eigenvalues) 


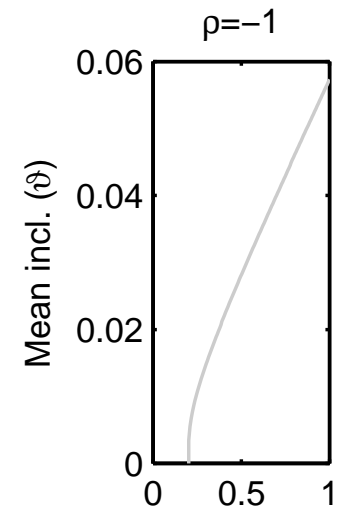

Circ. action $(\varphi)$
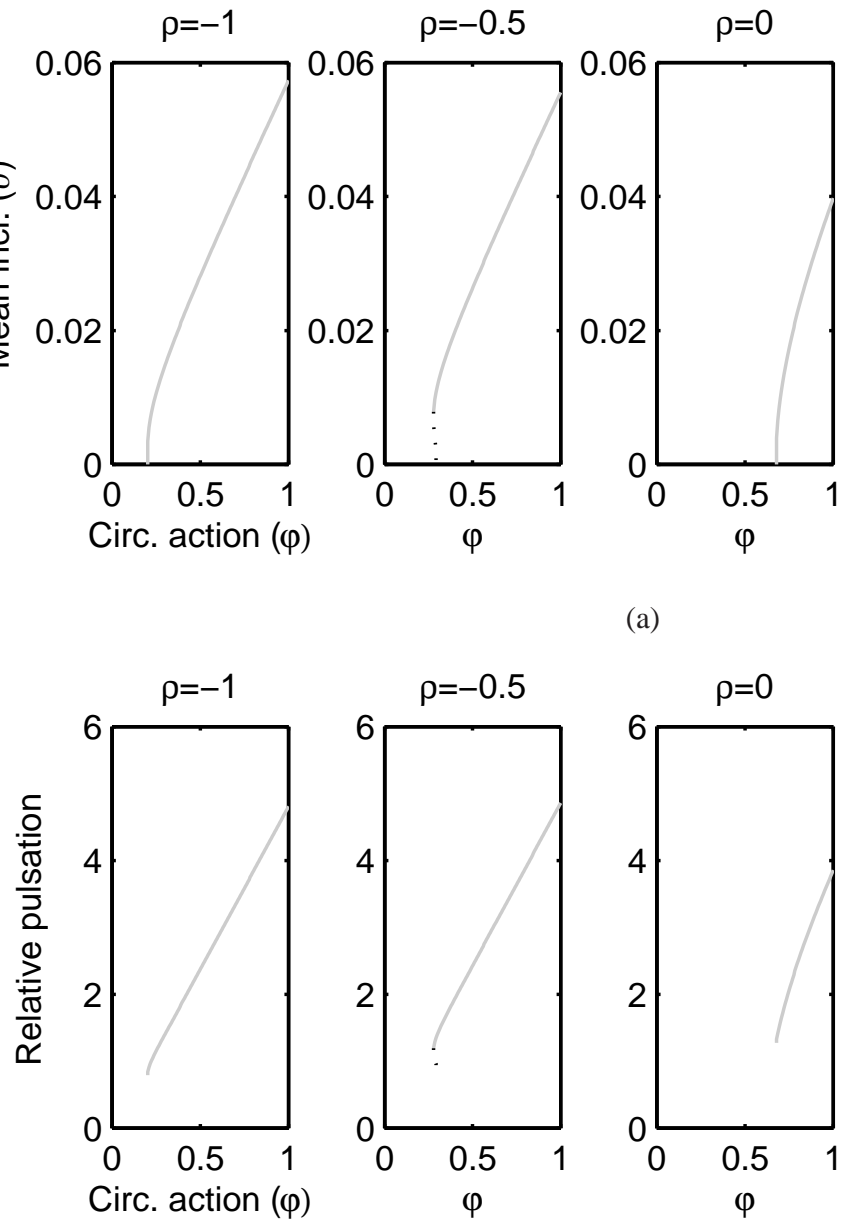

(a)
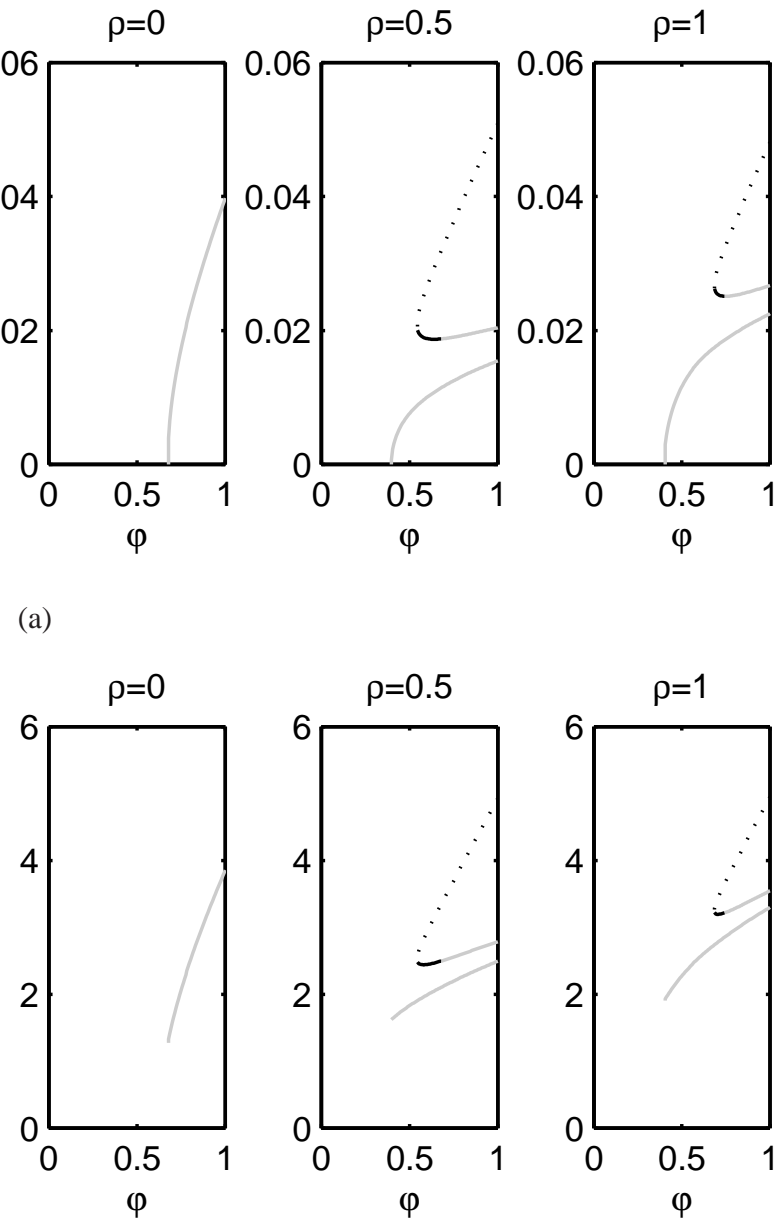

(b)

Figure 16: Limit cycles versus the circulatory action for various values of the gyroscopic action, for Eq. 1 with $\alpha=1.5, \alpha \beta=1, \xi_{t}=20 \%$ and $\sigma=10^{4}$, (a) mean inclination, (b) Relative frequency (gray: stable limit cycle, dot black: dominant real unstable eigenvalue, solid black: dominant pair of conjugate complex unstable eigenvalues) 


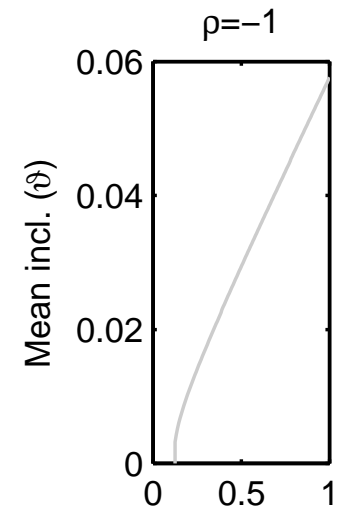

Circ. action $(\varphi)$
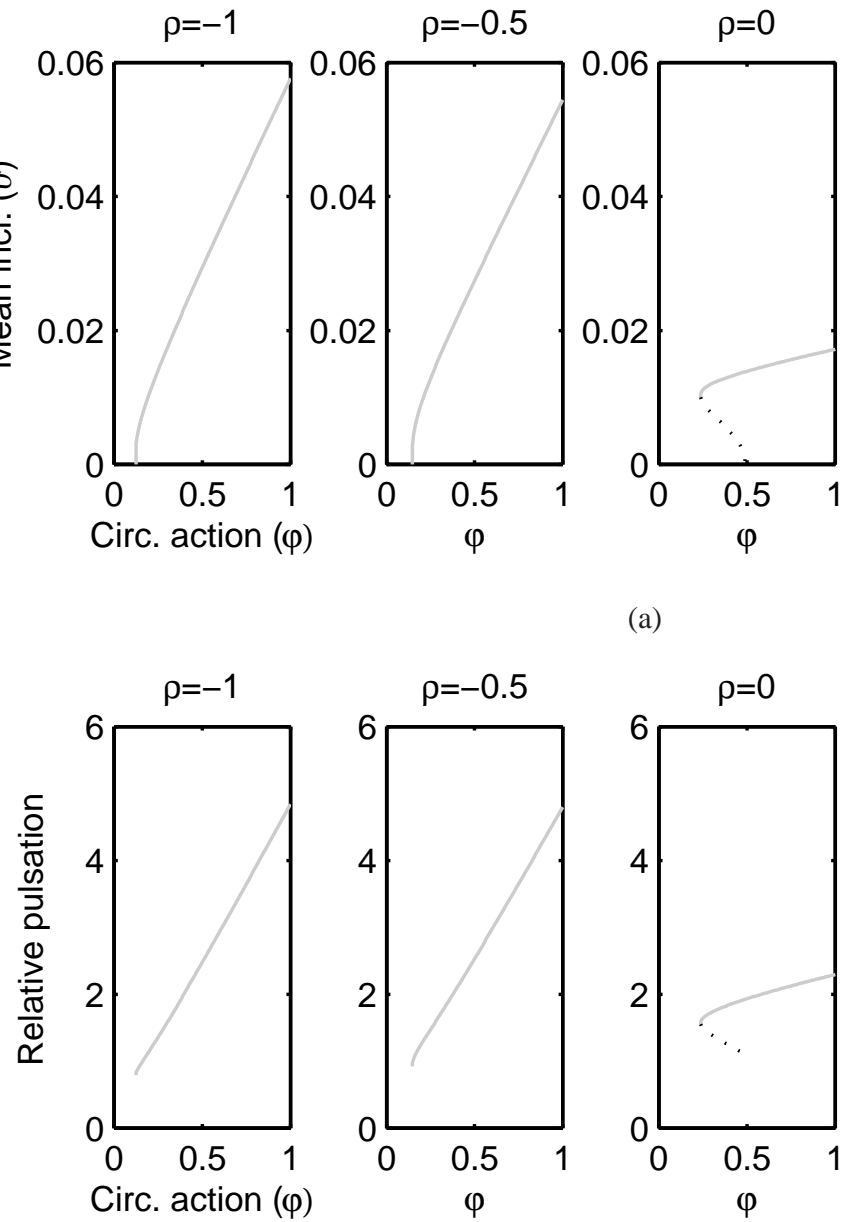

(a)
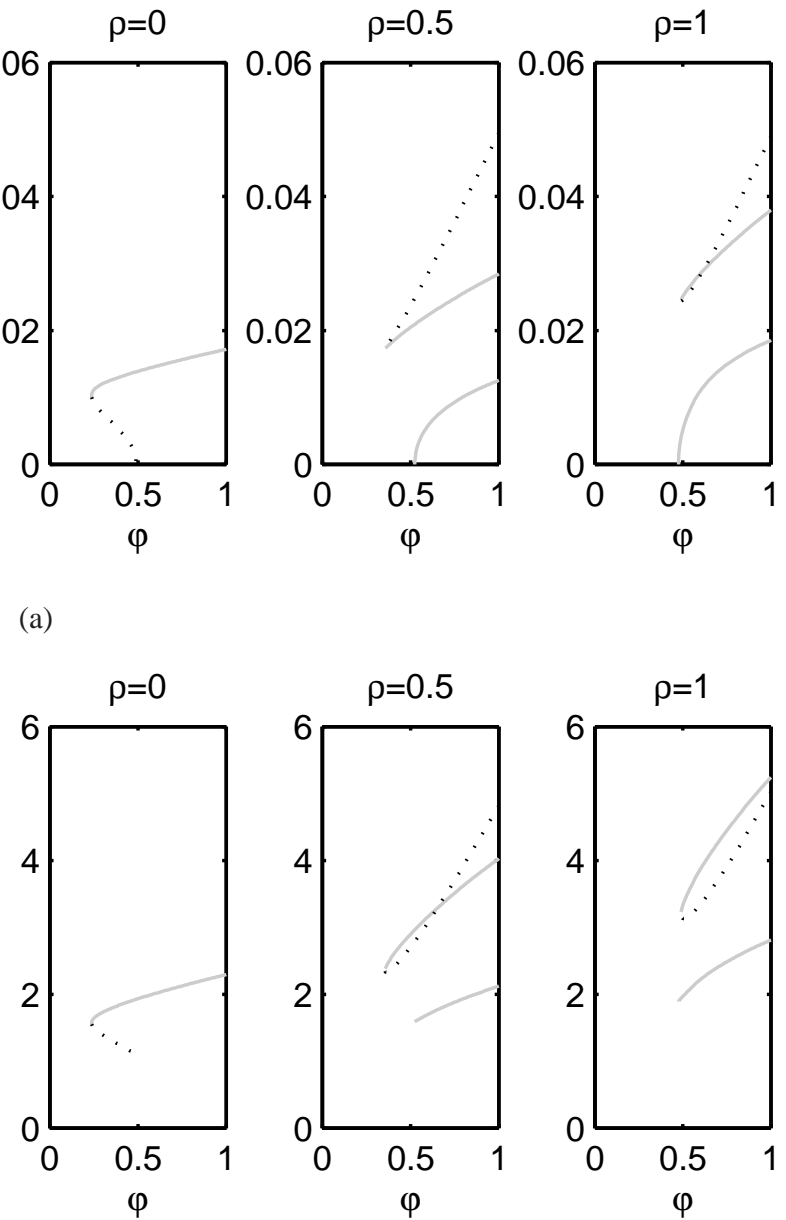

(b)

Figure 17: Limit cycles versus the circulatory action for various values of the gyroscopic action, for Eq. 1 with $\alpha=1.5, \alpha \beta=5, \xi_{t}=20 \%$ and $\sigma=10^{4}$, (a) mean inclination, (b) Relative frequency (gray: stable limit cycle, dot black: dominant real unstable eigenvalue, solid black: dominant pair of conjugate complex unstable eigenvalues) 

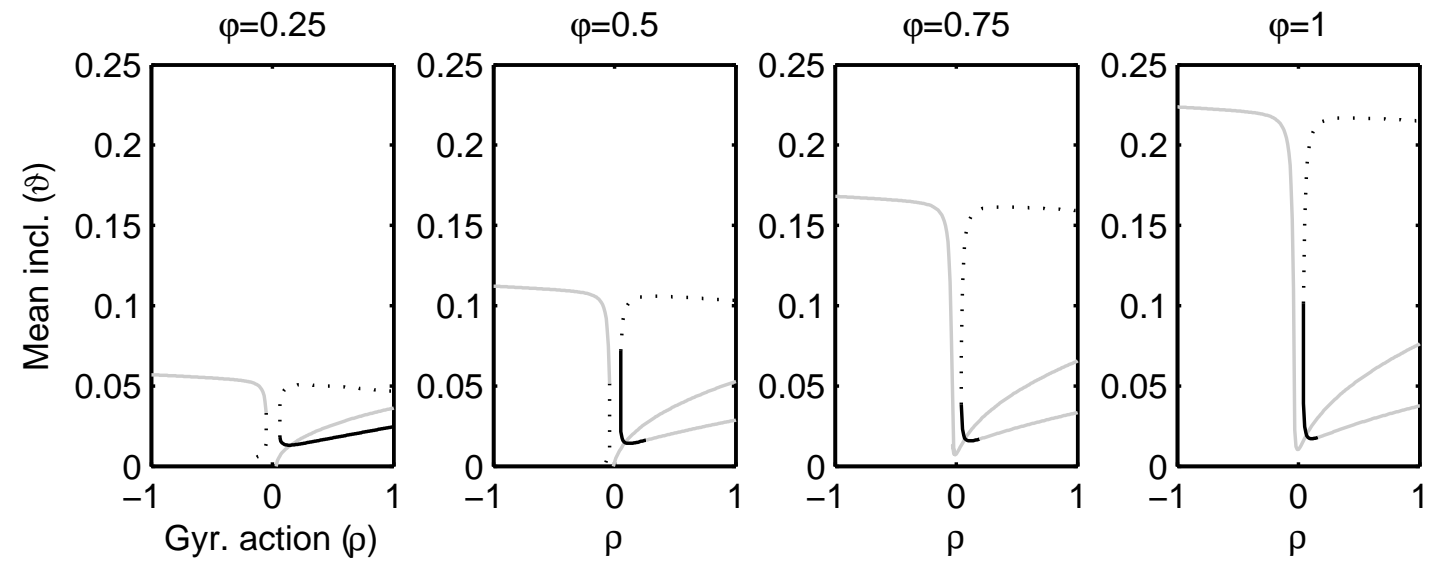

(a)
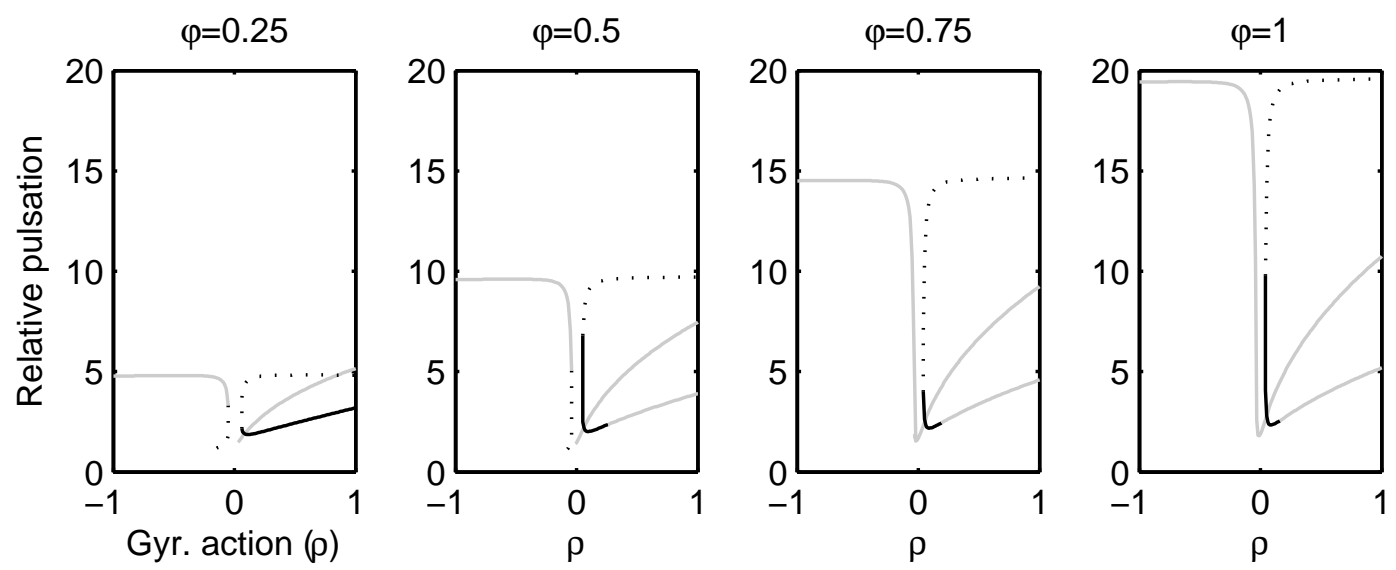

(b)

Figure 18: Limit cycles versus the gyroscopic action for various values of the circulatory action for Eq. 1 with $\alpha=1.5, \alpha \beta=0.2, \xi_{t}=5 \%$ and $\sigma=10^{4}$, (a) mean inclination, (b) Relative frequency (gray: stable limit cycle, dot black: dominant real unstable eigenvalue, solid black: dominant pair of conjugate complex unstable eigenvalues) 

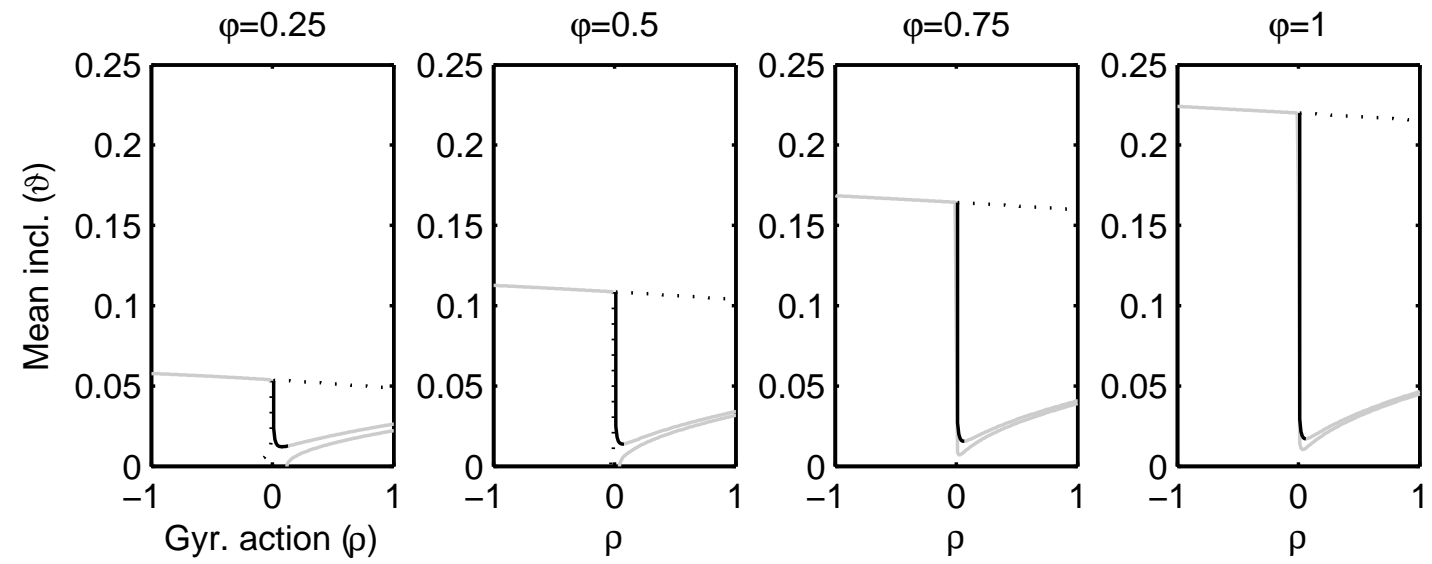

(a)
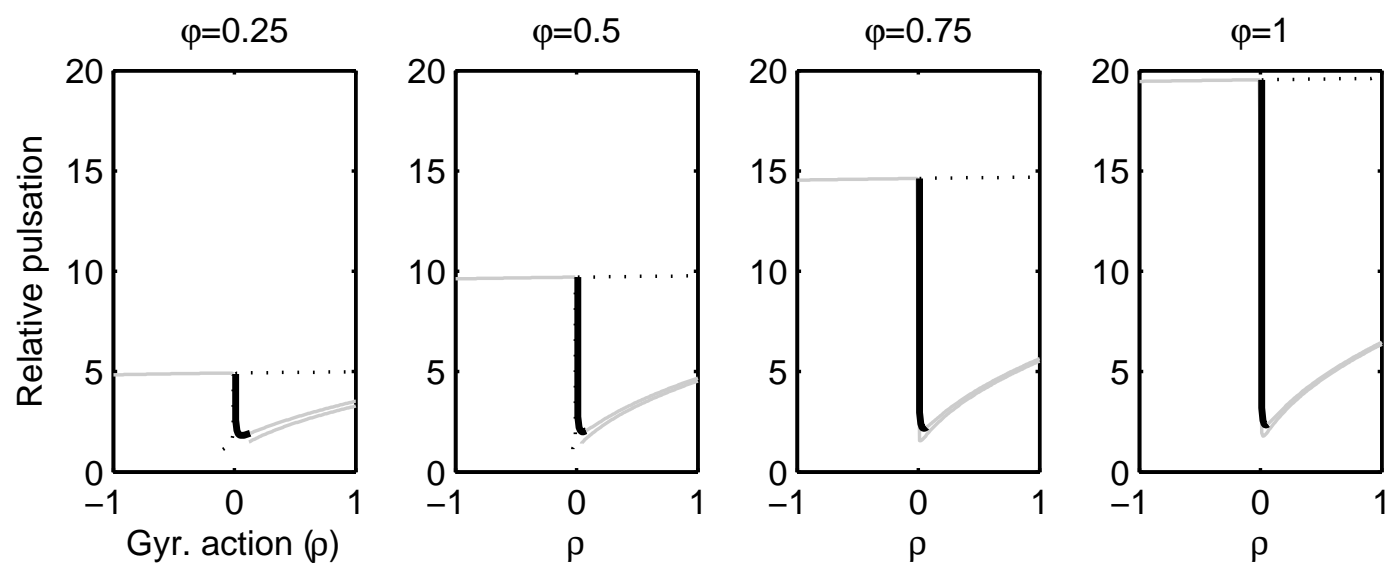

(b)

Figure 19: Limit cycles versus the gyroscopic action for various values of the circulatory action for Eq. 1 with $\alpha=1.5, \alpha \beta=1, \xi_{t}=5 \%$ and $\sigma=10^{4}$, (a) mean inclination, (b) Relative frequency (gray: stable limit cycle, dot black: dominant real unstable eigenvalue, solid black: dominant pair of conjugate complex unstable eigenvalues) 

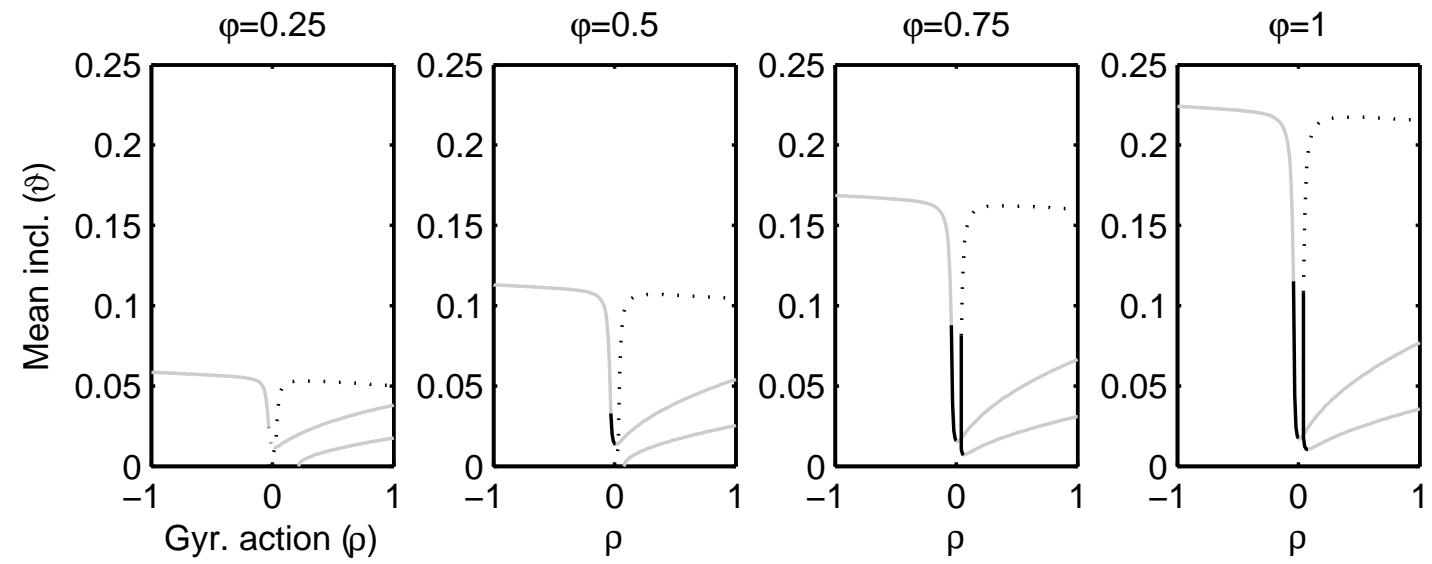

(a)
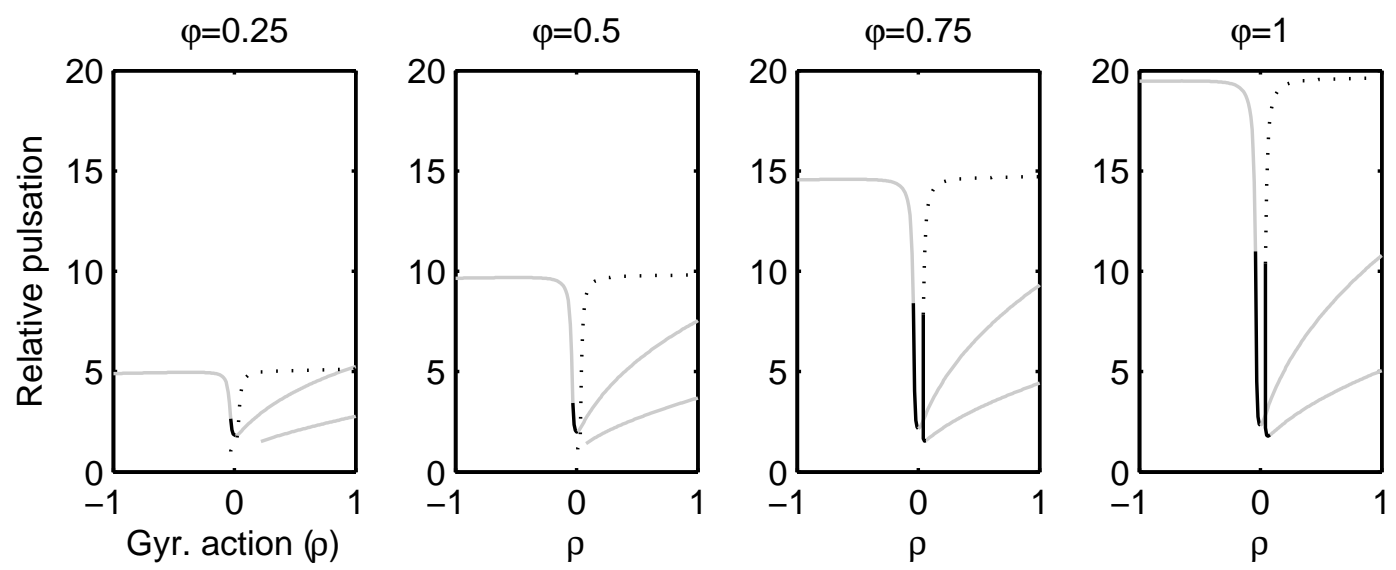

(b)

Figure 20: Limit cycles versus the gyroscopic action for various values of the circulatory action for Eq. 1 with $\alpha=1.5, \alpha \beta=5, \xi_{t}=5 \%$ and $\sigma=10^{4}$, (a) mean inclination, (b) Relative frequency (gray: stable limit cycle, dot black: dominant real unstable eigenvalue, solid black: dominant pair of conjugate complex unstable eigenvalues) 

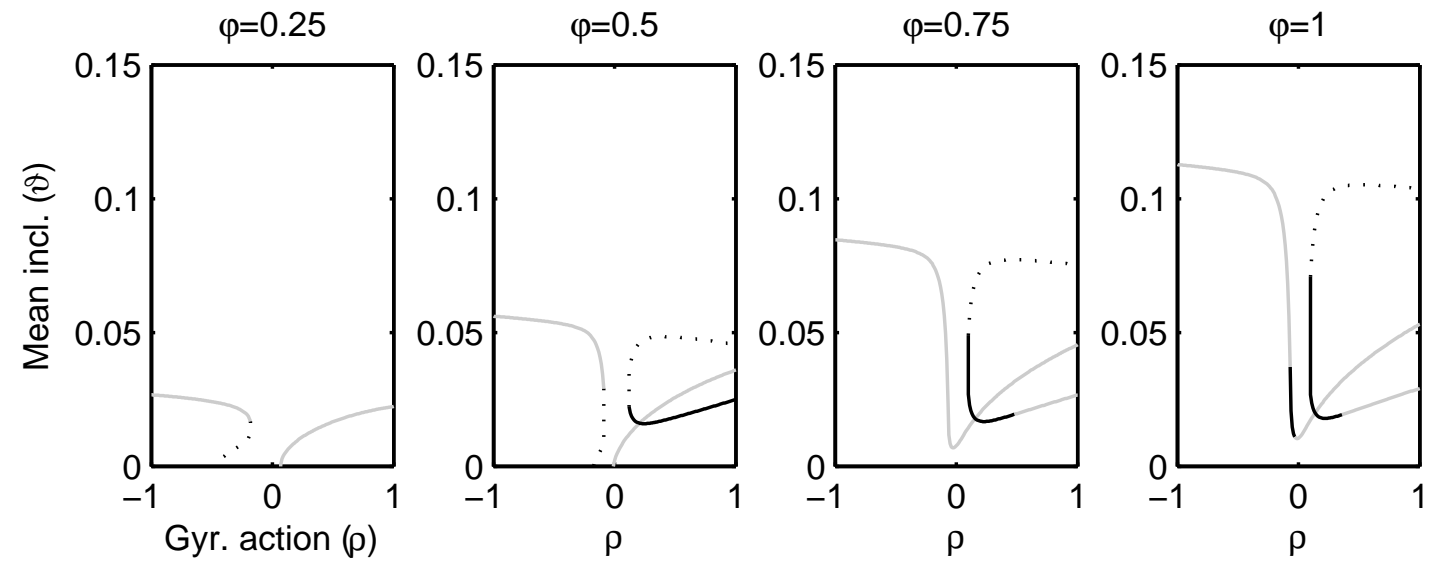

(a)
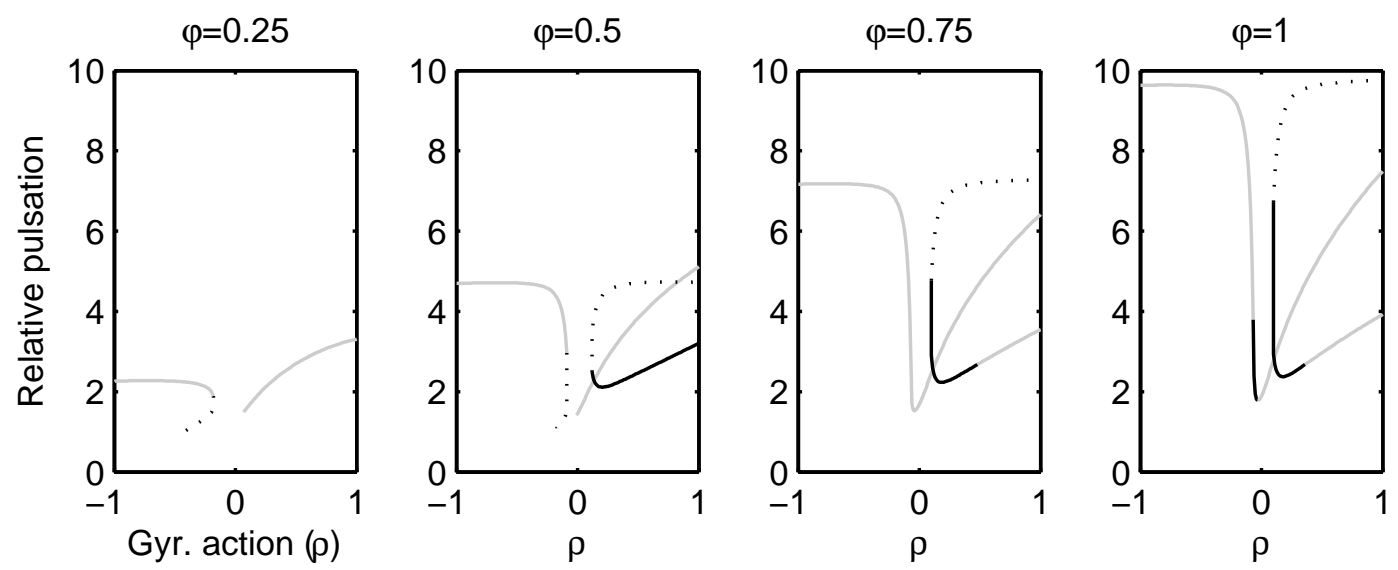

(b)

Figure 21: Limit cycles versus the gyroscopic action for various values of the circulatory action for Eq. 1 with $\alpha=1.5, \alpha \beta=0.2, \xi_{t}=10 \%$ and $\sigma=10^{4}$, (a) mean inclination, (b) Relative frequency (gray: stable limit cycle, dot black: dominant real unstable eigenvalue, solid black: dominant pair of conjugate complex unstable eigenvalues) 

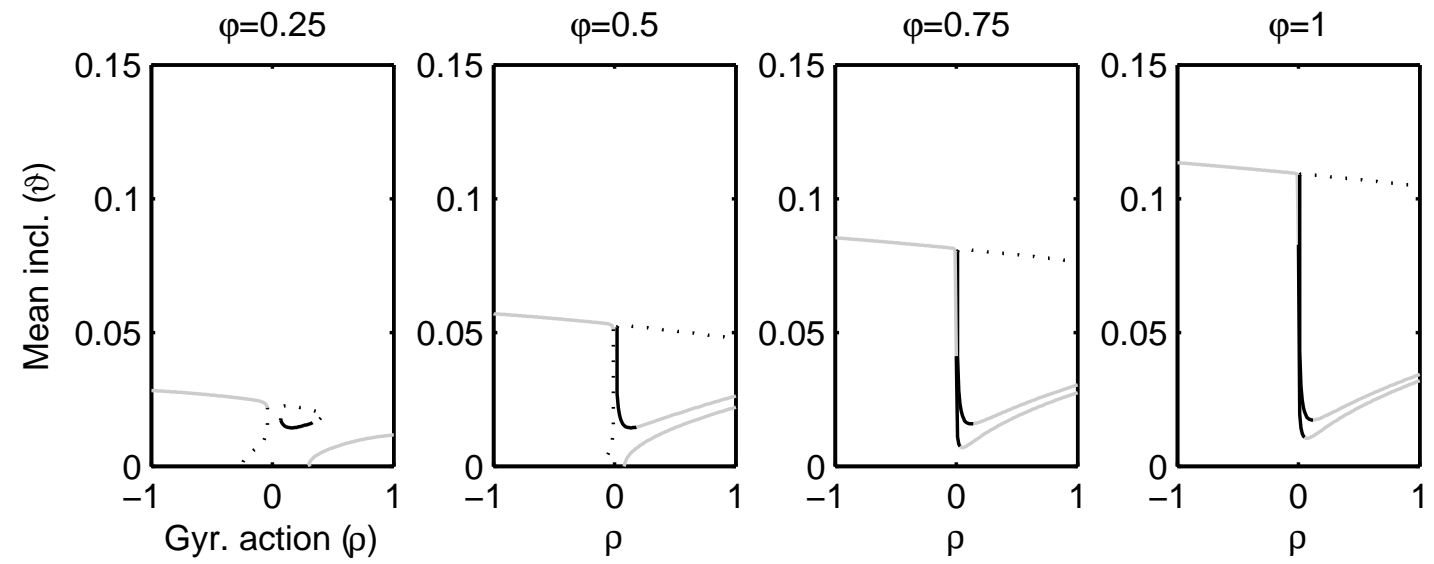

(a)
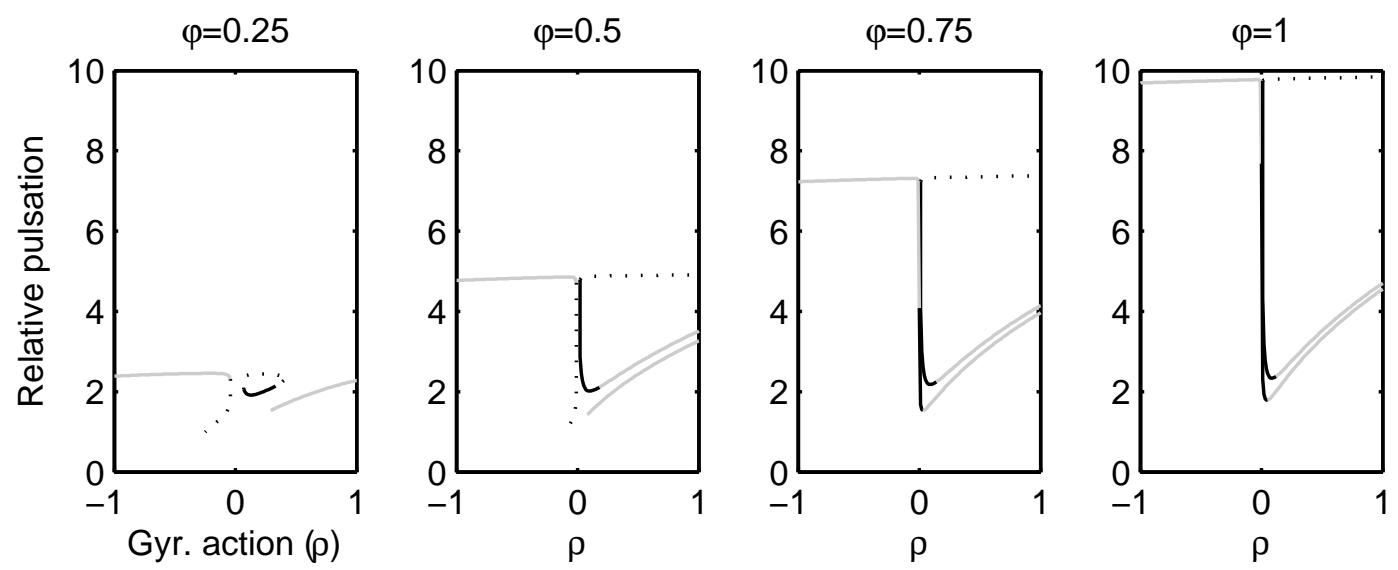

(b)

Figure 22: Limit cycles versus the gyroscopic action for various values of the circulatory action for Eq. 1 with $\alpha=1.5, \alpha \beta=1, \xi_{t}=10 \%$ and $\sigma=10^{4}$, (a) mean inclination, (b) Relative frequency (gray: stable limit cycle, dot black: dominant real unstable eigenvalue, solid black: dominant pair of conjugate complex unstable eigenvalues) 

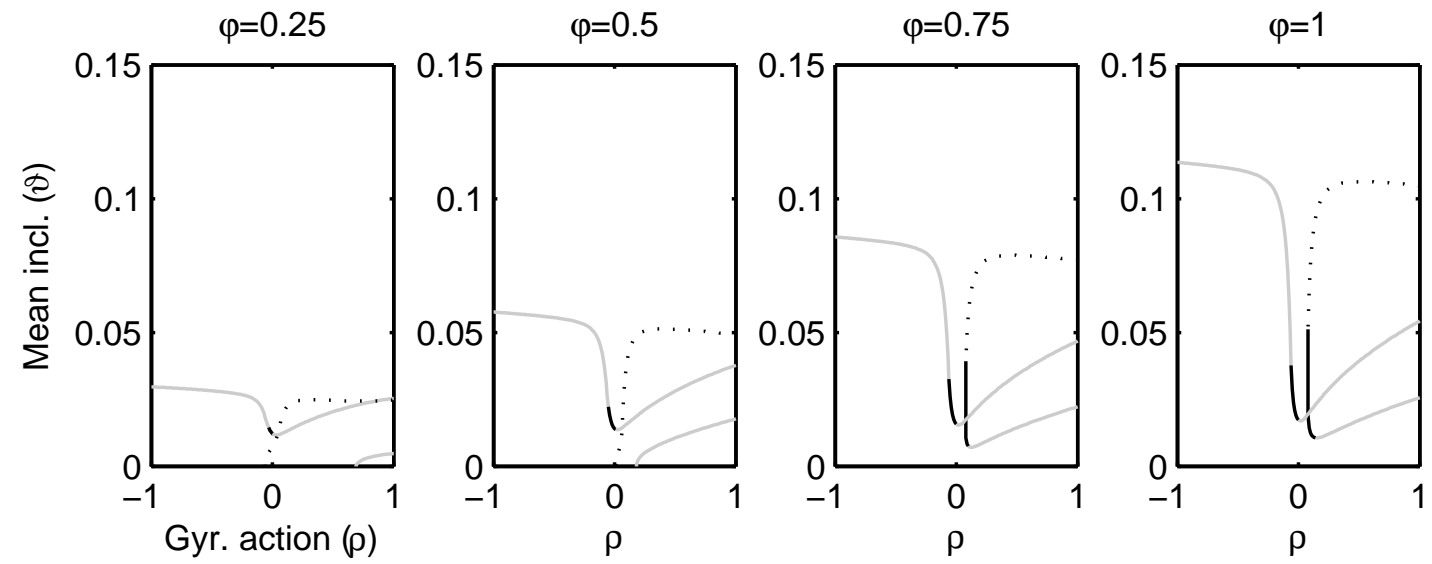

(a)
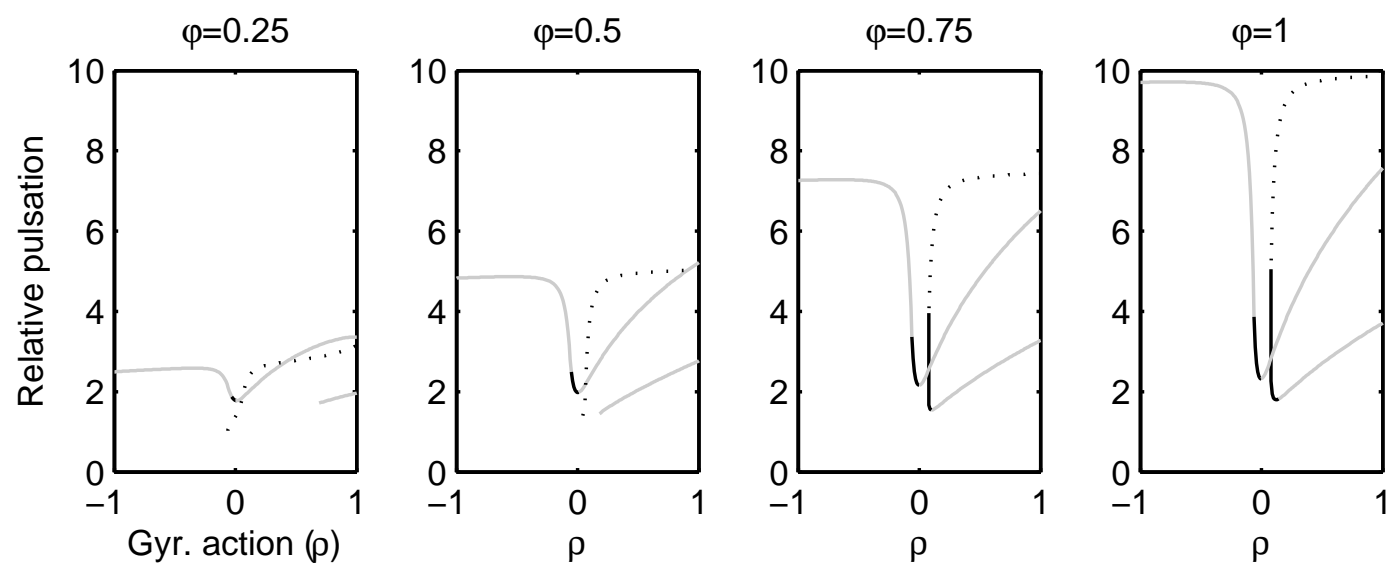

(b)

Figure 23: Limit cycles versus the gyroscopic action for various values of the circulatory action for Eq. 1 with $\alpha=1.5, \alpha \beta=5, \xi_{t}=10 \%$ and $\sigma=10^{4}$, (a) mean inclination, (b) Relative frequency (gray: stable limit cycle, dot black: dominant real unstable eigenvalue, solid black: dominant pair of conjugate complex unstable eigenvalues) 

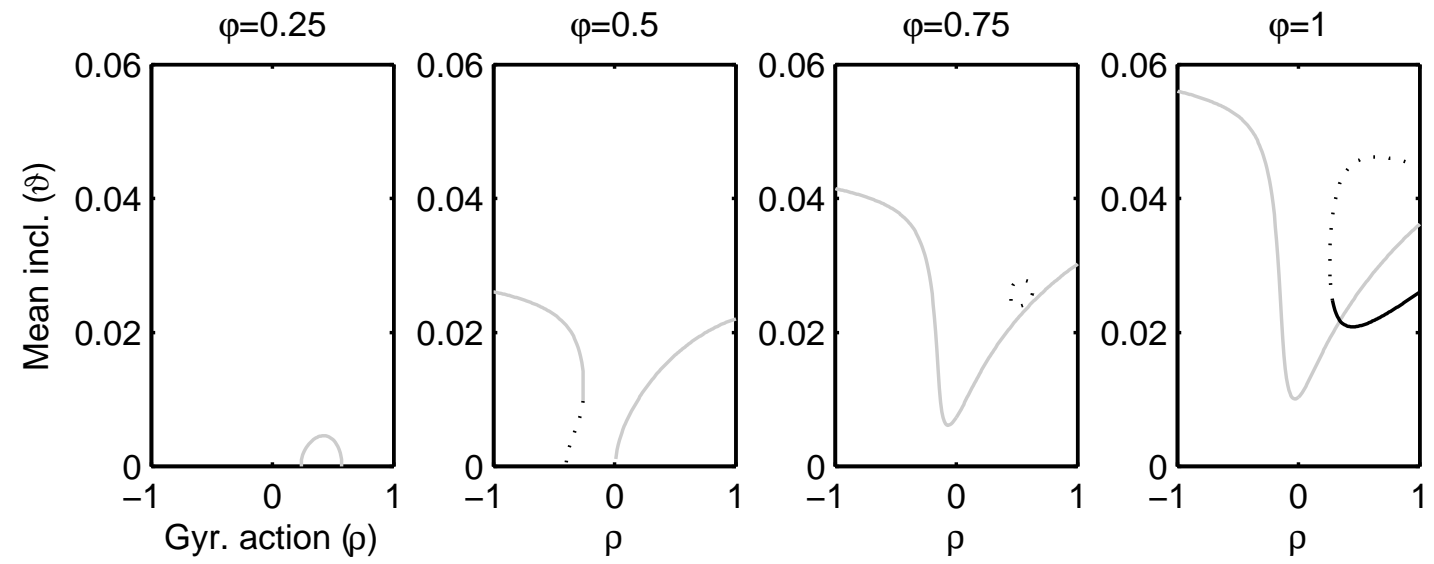

(a)
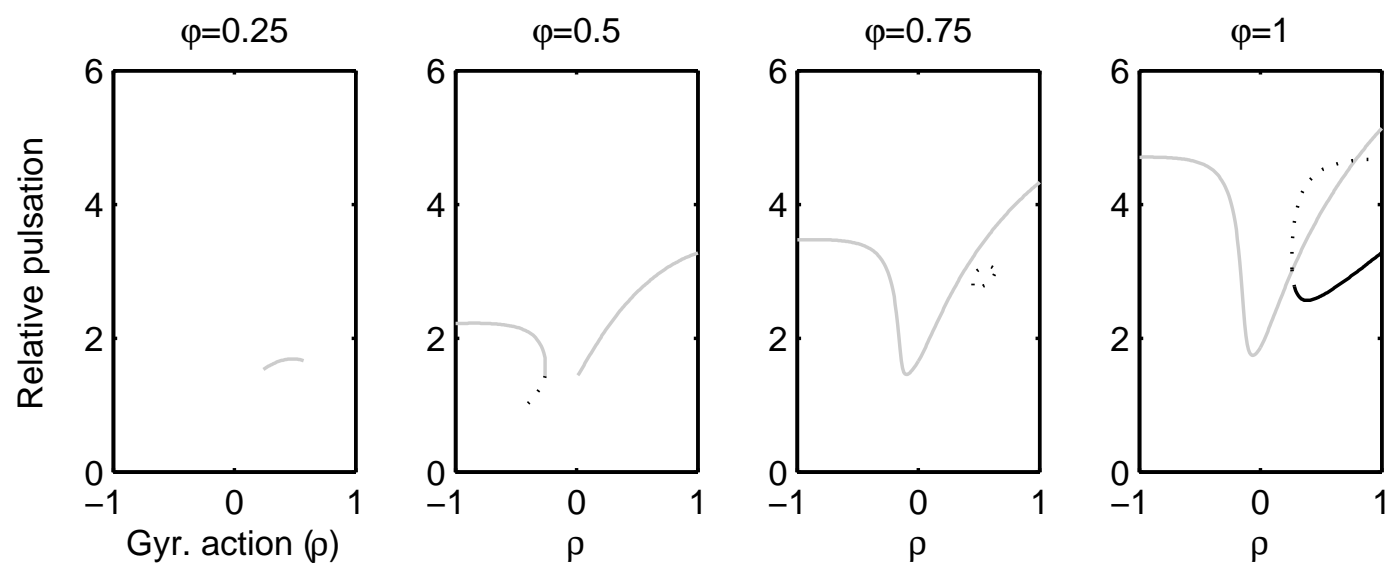

(b)

Figure 24: Limit cycles versus the gyroscopic action for various values of the circulatory action for Eq. 1 with $\alpha=1.5, \alpha \beta=0.2, \xi_{t}=20 \%$ and $\sigma=10^{4}$, (a) mean inclination, (b) Relative frequency (gray: stable limit cycle, dot black: dominant real unstable eigenvalue, solid black: dominant pair of conjugate complex unstable eigenvalues) 

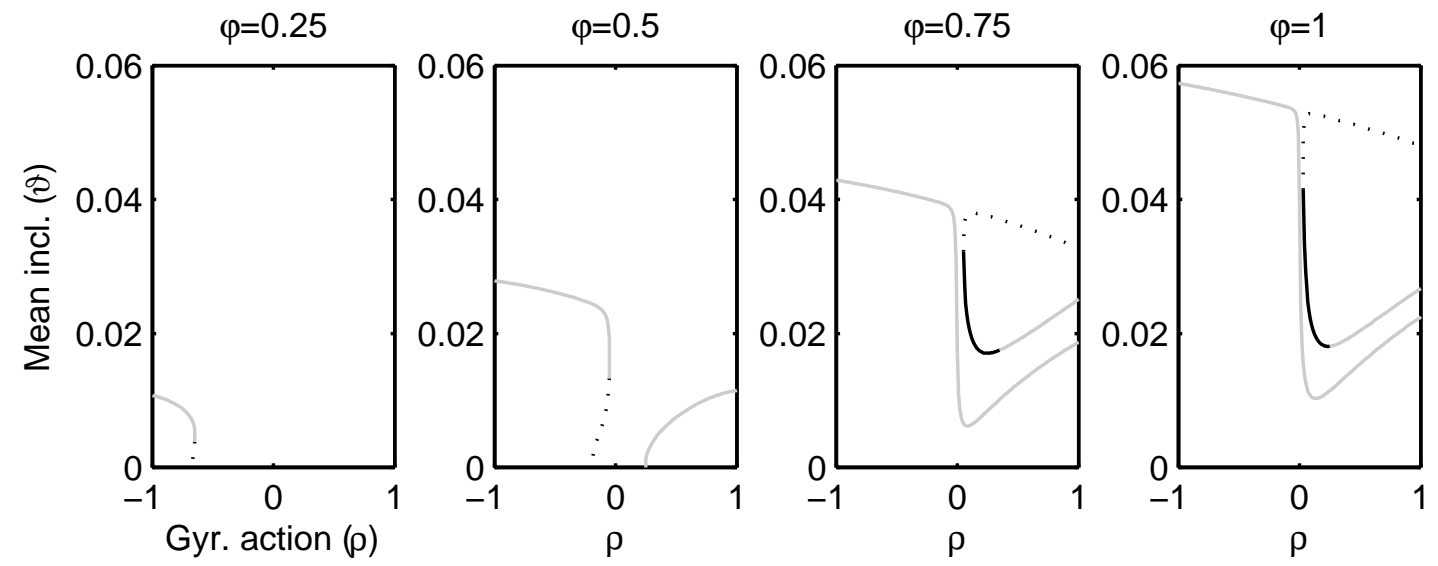

(a)
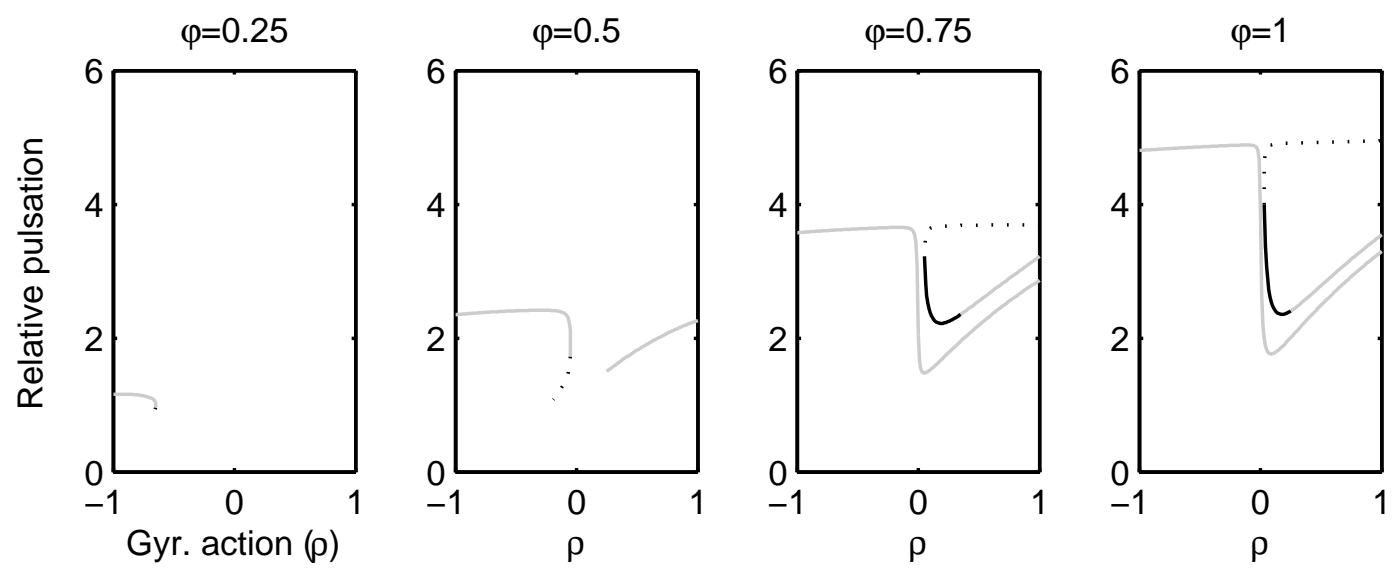

(b)

Figure 25: Limit cycles versus the gyroscopic action for various values of the circulatory action for Eq. 1 with $\alpha=1.5, \alpha \beta=1, \xi_{t}=20 \%$ and $\sigma=10^{4}$, (a) mean inclination, (b) Relative frequency (gray: stable limit cycle, dot black: dominant real unstable eigenvalue, solid black: dominant pair of conjugate complex unstable eigenvalues) 

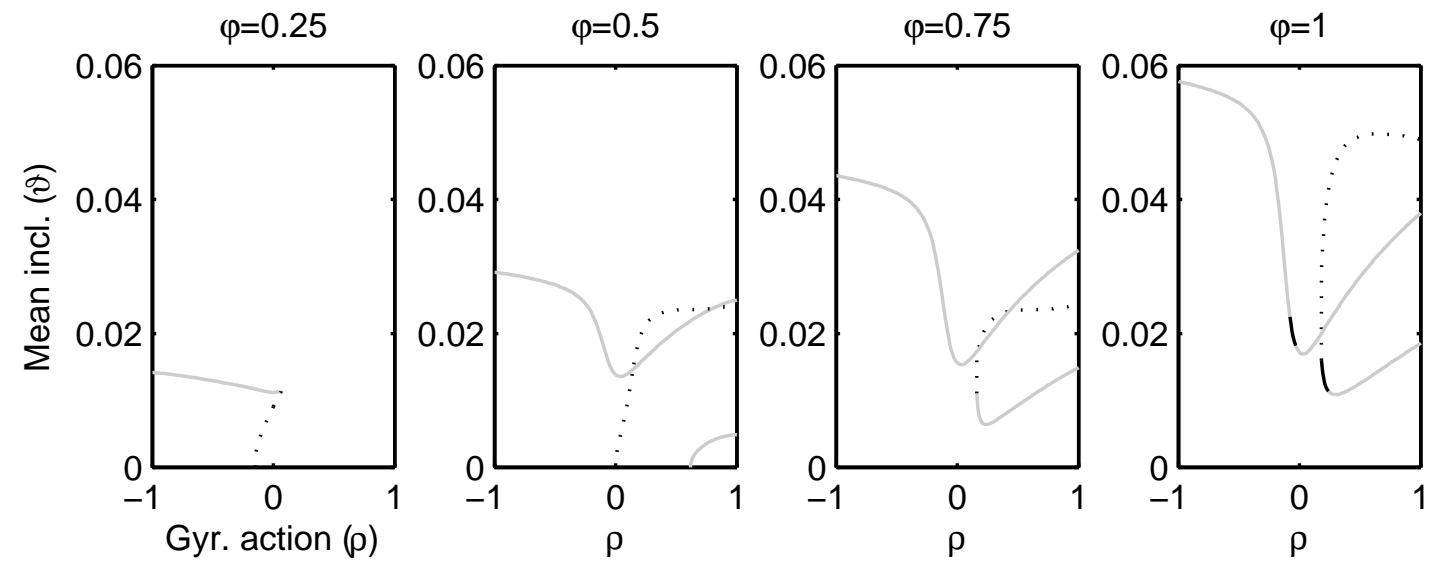

(a)
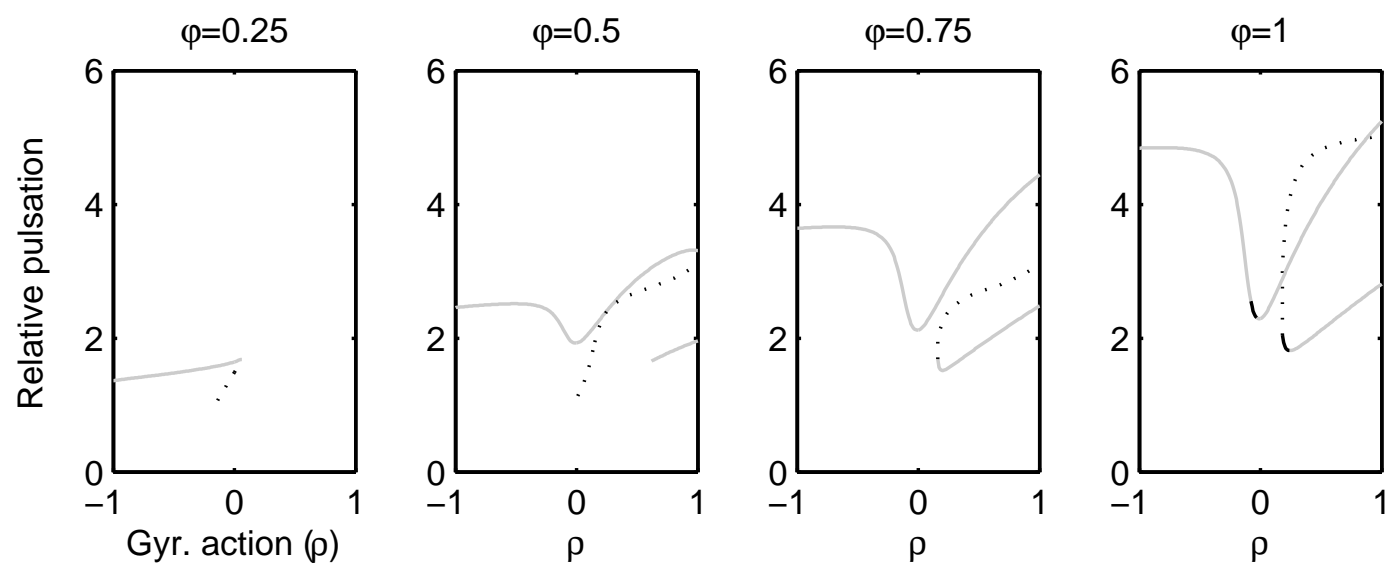

(b)

Figure 26: Limit cycles versus the gyroscopic action for various values of the circulatory action for Eq. 1 with $\alpha=1.5, \alpha \beta=5, \xi_{t}=20 \%$ and $\sigma=10^{4}$, (a) mean inclination, (b) Relative frequency (gray: stable limit cycle, dot black: dominant real unstable eigenvalue, solid black: dominant pair of conjugate complex unstable eigenvalues) 
jump phenomena whereas a positive gyroscopic action leads to a supercritical bifurcation. The additional branch appears for a large enough circulatory action.

The fast evolution of the limit cycle which is observed in the vicinity of the purely circulatory system for $\alpha \beta=1$ is shifted to negative values of the gyroscopic action as $\alpha \beta$ is shifted to $\alpha \beta<1$. As a consequence, the purely circulatory system shows a far lower limit cycle amplitude and frequency and a very low sensitivity to the gyroscopic action, see Fig. 18,21 and 24. The possibility to reach a minimum of amplitude and minimum of sensitivity with regard to the gyroscopic action by varying the damping distribution is suggested by this result. In the meanwhile, the additional limit cycle branch is shifted to positive values of the gyroscopic action and positive values of the circulatory action. The amplitude and frequency of its lowest amplitude part gets separated from the first branch and their growth is slower with regard to both the circulatory and the gyroscopic actions, whereas the first branch shows a faster growth. Nevertheless, apart from these points and the modification of the stability frontier of the equilibrium, the limit cycle branches look very similar for $\alpha \beta=1$ and $\alpha \beta<1$.

Contrariwise, the behaviour of the limit cycle branches becomes more complex as $\alpha \beta$ is shifted to $\alpha \beta>$ 1. Fig. 20, 23 and 26 reveal that all the parts of the branches have changed their connections in the vicinity of the purely circulatory system and now all branches interconnect. On Fig. 20, 23 and 26, it can be observed that the limit cycles are unstable by complex eigenvalues of the monodromy matrix where the parts connect. Moreover, a particular effect can be observed on Fig. 20, 23 and 26 for the purely circulatory system which now shows a subcritical bifurcation. A subcritical bifurcation with no stable limit cycle is even observed for the lowest values of the damping amount, see Fig. 11 and 14. As a consequence when the circulatory action crosses the stability frontier a jump phenomenon to a steady state which is not a limit cycle is obtained.

Another consequence of the interconnection between the limit cycle branches is that the steady state of the system can depend on the time history of its parameters, independently from considerations about initial conditions. Indeed, assume the dynamics of the system remains always close to the identified limit cycles for slowly varying parameters and follows their branches by continuity. As an example, consider Fig. 17 and 26. Assume a negative gyroscopic action, e.g. $\rho=-1$, and a slowly increasing circulatory action, from $\varphi=0$ to $\varphi=1$. The system shows a unique stable limit cycle as the equilibrium destabilizes. Moreover, no other stable solution could be identified, thus the steady state is not conditioned by the initial conditions. Then, assume $\varphi$ is kept constant and $\rho$ varies from $\rho=-1$ to $\rho=1$. Then, the arrival state is different of that reached when varying $\varphi$ from $\varphi=0$ to $\varphi=1$ with $\rho=1$ kept constant. A jump phenomena between limit cycles corresponding to a change of branch can even be expected in the later case if $\rho$ is then decreased from $\rho=1$ to $\rho=-1$.

To summarize, some general tendencies can be extracted from this parametric study on the role of the damping structure, the coupling actions and the nonlinearity.

- The stiffening rate of the system for non-vanishing motions ( $\sigma$ in Eq. 1) has an independent and strong influence as previously mentioned, the more stiffening the lower amplitude but without any effect on the frequency.

- The damping amount has a rather independent effect too, the more damping the lower amplitude and frequency.

- The circulatory action shows a general tendency to increase both the amplitude and the frequency.

- The non-symmetrical dependency of the limit cycle to either positive or negative gyroscopic action or damping distribution with $\alpha \beta<1$ or $\alpha \beta>1$ was highlighted.

- For the purely circulatory system with an iso-distribution of damping, a high sensitivity to the gyroscopic action is observed. Changing the damping distribution to either $\alpha \beta<1$ or $\alpha \beta>1$ alters this phenomenon by shifting the fast evolution front to negative values of the gyroscopic action so that the amplitude and frequency can be largely reduced for the purely circulatory system. 


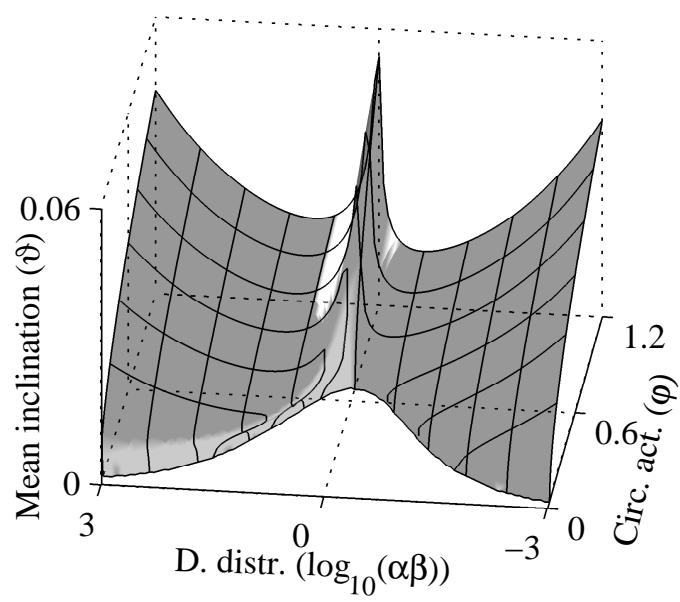

(a)

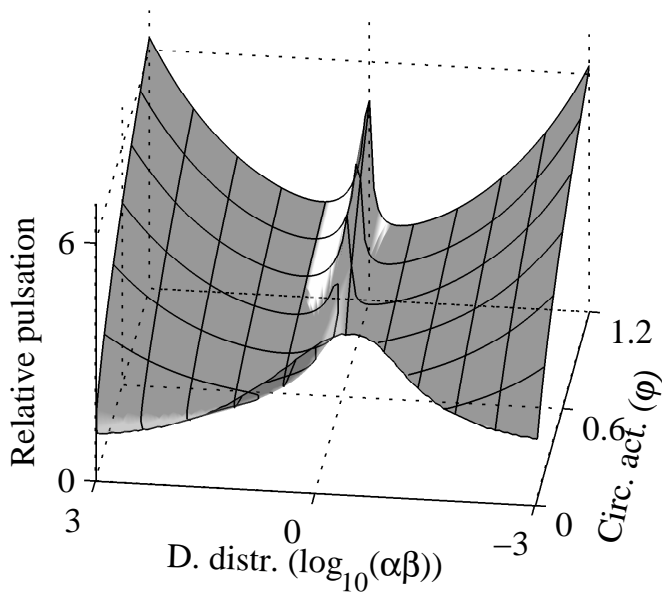

(b)

Figure 27: Limit cycle of the purely circulatory system versus the damping distribution and the circulatory action for Eq. 1 with $\alpha=1.5, \xi_{t}=20 \%$ and $\sigma=10^{4}$ (black: stable limit cycle, gray: dominant real unstable eigenvalue, white: dominant pair of conjugate complex unstable eigenvalues)

It was also conjectured the possibility to reach a robust minimum of amplitude and frequency by varying the damping distribution for the purely circulatory system. As a matter of fact, the iso-distribution of damping seems to maximize the amplitude and frequency. This particular property is emphasized on Fig. 27 which shows the evolution of the unique limit cycle identified for the purely circulatory system over a range of damping distribution.

It appears clearly on this figure that the iso-distribution leads to a faster increase of the amplitude and frequency with regard to the circulatory action and represents a local maximum far from the stability frontier. Contrariwise, local minima can be observed for either $\alpha \beta<1$ or $\alpha \beta>1$. The bifurcation nature changes from supercritical to subcritical as $\alpha \beta$ changes from $\alpha \beta<1$ to $\alpha \beta>1$. Thus not only avoiding an iso-distribution of damping can allow reducing the limit cycle amplitude but $\alpha \beta<1$ also appears more favourable than $\alpha \beta>1$ because of the nature of the associated bifurcation. As an important consequence, a proper design strategy can allow reducing the amplitude of self-generated vibrations and avoiding subcritical bifurcations in the same time by adjusting the damping distribution. It was shown in the stability analysis of the equilibrium that the widest stability domain is found near the iso-distribution of damping $(\alpha \beta=1)$. Therefore, in a small enough vicinity of the destabilization point, the iso-distribution of damping can appear as a local minimum of amplitude because of the late rise of the limit cycle. However, this situation reverses far from the destabilization point and the iso-distribution of damping becomes a local maximum of the amplitude. In order to explain this observation, it can be reminded that for the purely circulatory system with an iso-distribution of damping, both stable and unstable modes have the same frequency (see [13]). Thus, an intrinsic resonance of the stable mode can be evocated to explain this particular behaviour.

Therefore, the iso-distribution of damping restrains the most the purely circulatory system from destabilizing but to the detriment of the amplitude of the dynamic state that follows the destabilization. This can be considered as a dynamic extension of the destabilization paradox. As a concluding remark, the important contrast between the results from the stability analysis of the equilibrium and the limit cycle amplitude regarding the influence of the parameters highlights the large difference which exists between a linear and a nonlinear investigation of the system. These two viewpoints are complementary and require an equal attention in order to define relevant design strategies. Countermeasures to self-generated vibrations based exclusively on a stability analysis of the equilibrium would suggest an iso-distribution of 
damping which can lead to even higher vibrations amplitude. Contrariwise, taking into account the limit cycles investigation allows defining two distinct approaches depending on whether or not the instability can be retained.

\section{Some comments on the nonperiodic dynamic behavior}

In some circumstances neither a stable equilibrium nor a stable limit cycle can be found, as an example see Fig. 23 for $\varphi=1$ and $\rho \in[-0.01,-0.05]$. This situation can not be highlighted by the sole identification of the limit cycle with the proposed method. The stability analysis of the estimated solution is also necessary. Indeed, a multi-DOF second order system can admit steady solutions which are neither equilibrium nor limit cycles, but dense trajectories in the state space, strange attractors or chaotic motions. In the present section, it is proposed to illustrate such dynamic behaviours for the proposed system. A practical way to do so is to use Poincaré sections, see [18].

In the state space, assume a lower dimensional subspace crossed by the studied trajectory, called a Poincare section. Then, the locus of the recursive crossings of this subspace by the trajectory in a chosen direction forms the Poincare application and is intimately related to the nature of the motion.

Such sections are reported on Fig. 28 for various values of the gyroscopic action and the same values of the other parameters as on Fig. 23.

Depending on the gyroscopic action, the Poincaré section shows various pattern.

- For $\rho=0$, the Poincaré section is restricted to a unique point, which indicates a periodic motion, i.e. a limit cycle, confirmed by the time evolution of the variables.

- For $\rho=-0.01$, the Poincaré section is a single loop closed curve, corresponding to the section of a torus in the state space, thus the motion is biperiodic. The time evolution of the variables shows pseudo-periodic oscillations limited by a periodic envelop. Moreover, eigenvalues of the monodromy matrix of the identified limit cycle cross the unit circle through non-real values between $\rho=0$ and $\rho=-0.01$, thus a Neimark-Sacker bifurcation, i.e. between a limit cycle and a torus, see [19], can be conjectured to occur.

- For $\rho=-0.02$, the previous closed curve now appears divided into two imbricated loops, which can indicate a period doubling bifurcation of the secondary period of the torus between $\rho=-0.01$ and $\rho=-0.02$. This is qualitatively supported by the previously observed envelop being changed into a half-frequency new one.

- For $\rho=-0.03$ and $\rho=-0.04$, the Poincaré section show scattered points in the vicinity of the previous closed curve and the corresponding motion is rather irregular. A bifurcation to a $n$ periodic motion with $n \geq 3$, a strange attractor or a chaotic attractor according to the Ruelle-Takens scenario can be evocated, see [21].

- For $\rho=-0.05$, the Poincaré changes back to a closed curve, indicating a biperiodic motion.

- Finally, for $\rho=-0.06$ the Poincaré section is restricted again to a unique point, which indicates the stabilization of the limit cycle.

By comparing Fig. 23 and 28, it can be observed that the mean amplitude and the mean pseudo-period of the non-periodic motions remain close to those of the identified (unstable) limit cycle. Therefore, even if the limit cycle is not the attractive state, its characteristics are representative of the steady motion and can still serve as a design criterion to some extend. This was also observed for other branches of unstable limit cycle.

In spite of the relative simplicity of the proposed model, a wide variety of behaviours is observed when both the equilibrium and the limit cycles destabilize. Therefore, one can presume that more detailed models with less regular nonlinearities are even more likely to show such a complexity. 

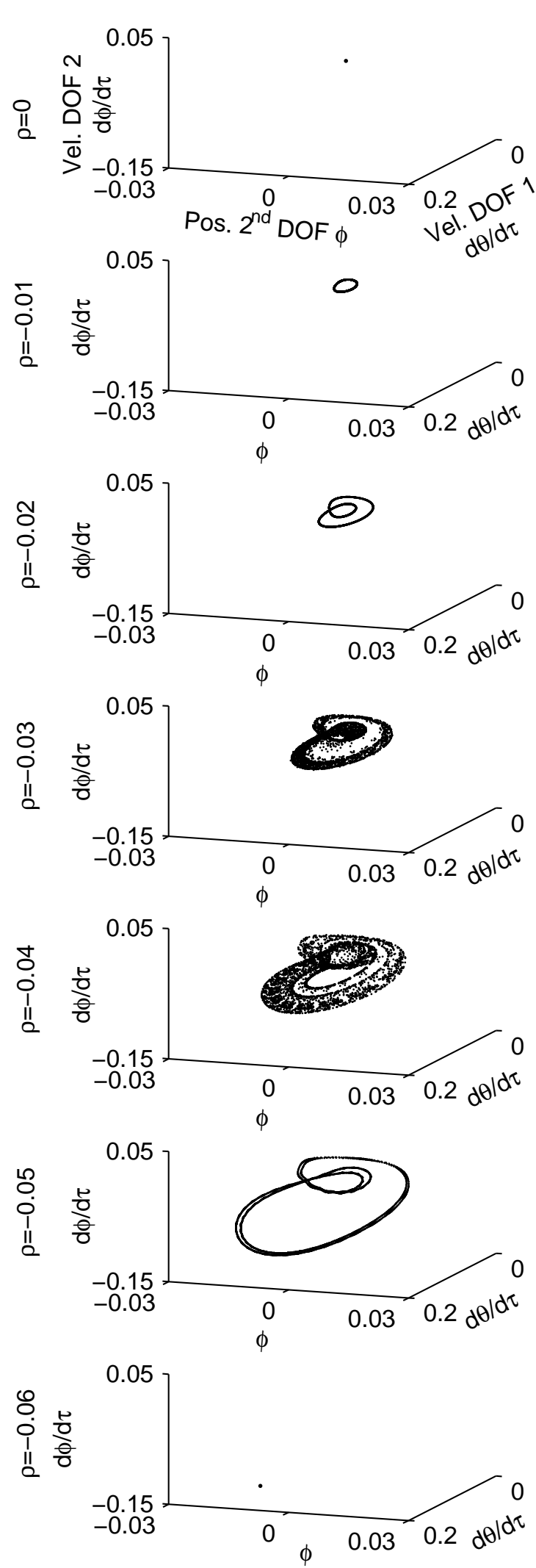
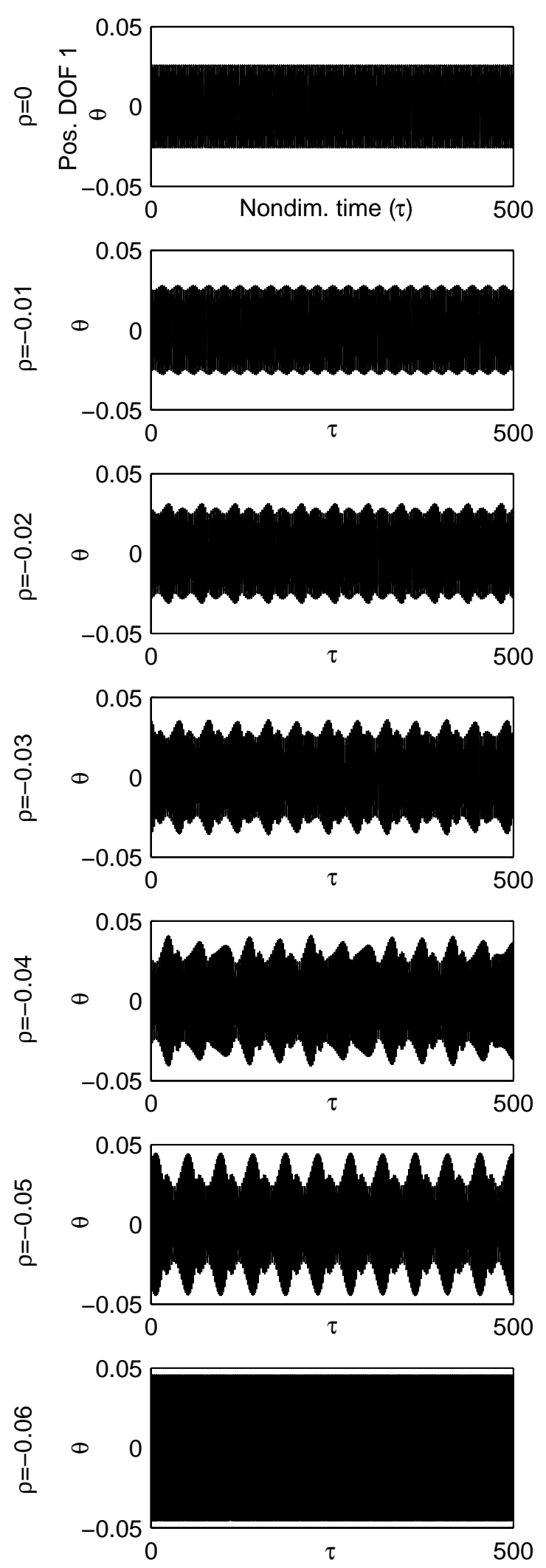

Figure 28: Steady solution of Eq. 1 with $\alpha=1.5, \alpha \beta=5, \xi_{t}=10 \%, \varphi=1, \sigma=10^{4}$ and various values of $\rho$, (a) Poincaré application in the section $\theta=0$ and $\dot{\theta}>0$, (b) position on the 1st DOF versus time 


\section{Conclusion}

A nonlinear 2-DOF system combining damping and both circulatory and gyroscopic actions has been extensively investigated. Regarding the equilibrium, an important effect of the damping structure on both the stability frontiers and the nature of the Hopf bifurcation has been exhibited. In particular, it appears that the iso-distribution of damping is nearly an optimum for the stability range of quasi-purely circulatory systems. The influence of the gyroscopic action has been illustrated too.

The identification of the limit cycles of the systems also revealed a particular influence of the damping distribution. Both the connections between the branches of limit cycles and their stability are affected. As an extension to the destabilization paradox, the iso-distribution of damping has been shown to induce the fastest growth of the limit cycles for quasi-purely circulatory systems. As a result, the iso-distribution of damping appears to be at the same time the most desirable structure in order to avoid the destabilization and the worst configuration in the purpose of reducing the amplitude of the self-generated vibrations. An internal resonance of the stable mode to the unstable mode is evocated to explain this particular behaviour. Moreover, the damping structure as well as the sign of the gyroscopic action play an important role in the nonlinear behaviour of the system. In particular, no symmetry can be observed on the branches of limit cycles between positive and negative values of the gyroscopic action in the proposed model. The influence of the damping structure shows no symmetry neither.

Finally, non-periodical stable motions have been shown to take place when neither stable equilibrium nor stable limit cycle were identified, including multi-periodic motion, strange or chaotic attractors. This large variety of behaviours exhibited for the rather simple autonomous 2-DOF lumped system allows emphasizing how complex the dynamics of real-life systems are likely to be. Although the proposed approach can be applied to some more sophisticated models, some major aspects of friction-induced selfgenerated vibrations are highlighted in this paper. Such minimalist models with few DOF can serve as effective tools for understanding the role of some physical parameters in identified coupling mechanisms.

\section{References}

[1] Kinkaid, N.M., O’Reilly, O.M., and Papadopoulos, P., 2003. “Automotive disc brake squeal”. Journal of Sound and Vibration, 267(1), pp. 105-166.

[2] Sinou, J.-J., Dereure, O., Mazet, G.-B., Thouverez, F., and Jézéquel, L., 2006. "Friction induced vibration for an aircraft brake system". International Journal of Mechanical Sciences, 48(5), pp. 536567.

[3] Ziegler, H., 1952. "Die Stabilitätskriterien der Elastomechanik". Ingenieur-Archiv, 20, p. 49-56.

[4] Bolotin, V.V., 1963. "Nonconservative problems of the theory of elastic stability". Oxford: Pergamon, translated by T.K. Lusher.

[5] Leipholz, H.H., 1980. "Stability of elastic systems". Sijthoff and Noordhoff, Alphen aan den Rijn.

[6] Herrmann, G., and Jong, I.C., 2007. "On nonconservative stability problems of elastic systems with slight damping". Journal of Applied Mechanics, 32, p. 125-133.

[7] O'Reilly, O.M., Malhotra, N.K., and Namachchivaya, N.S., 1996. "Some aspects of destabilization in reversible dynamical systems with application to follower forces". Nonlinear Dynamics, 10, p. 6387.

[8] Kirillov, O.N., and Seyranian, A.P., 2004. "Stabilization and destabilization of a circulatory system by small velocity-dependent forces”. Journal of sound and vibration, 283(3-5), p. 781-800.

[9] Kirillov, O.N., 2007. "Destabilization paradox due to breaking the Hamiltonian and reversible symmetry". International Journal of Non-linear Mechanics, 42(1), p. 71-87. 
[10] Hoffmann, N., and Gaul, L., 2003. "Effects of damping on mode-coupling instability in friction induced oscillations". Zeitschrift für Angewandte Mathematik und Mechanik, 83(8), pp. 524-534.

[11] Sinou, J.-J., and Jézéquel, L., 2007. "Mode coupling instability in friction-induced vibrations and its dependency on system parameters including damping". European Journal of Mechanics, A/Solids, 26(1), pp. 106-122.

[12] Fritz, G., Sinou, J.-J., Duffal, J.-M., and Jézéquel, L., 2007. "Investigation of the relationship between damping and mode-coupling patterns in case of brake squeal". Journal of Sound and Vibration, 307(3-5), p. 591-609.

[13] Hervé, B., Sinou, J.-J., Mahé, H., and Jézéquel, L., 2008. "Analysis of squeal noise and mode coupling instabilities including damping and gyroscopic effects". European Journal of Mechanics, A/Solids, 27(2), p. 141-160.

[14] Wickramarachi, P., Singh, R., and Bailey, G., 2005. "Analysis of friction-induced vibration leading to EEK noise in a dry friction clutch". Noise control engineering journal, 53(4), p. 138-144.

[15] Coudeyras, N., Sinou, J-J., and Nacivet, S., 2009. "A new treatment for predicting the selfexcited vibrations of nonlinear systems with frictional interfaces: the Constrained Harmonic Balance Method, with application to disc brake squeal". Journal of sound and vibration, 319, p. 1175-1199.

[16] Sotomayor, J., Mello, L.F., and Braga, D.C., 2007. "Bifurcation analysis of the Watt governor system". Computational and Applied Mathematics, 26(1), p. 19-44.

[17] Takens, F., 1973. "Unfolding of certain singularities of vector fields: Generalized Hopf bifurcations". Journal of Differential Equations, 14(3), p. 476-493.

[18] Guckenheimer, and J., Holmes, P., 1983. "Nonlinear Oscillations, Dynamical Systems and Bifurcation of Vector Fields". Springer-Verlag.

[19] Sacker, R., 1964. "On invariant surfaces and bifurcation of periodic solutions of ordinary differential equations". report IMM-NYU 333, New York University.

[20] Thomsen, J.J., 1995. "Chaotic dynamics of the partially follower-loaded elastic double pendulum". Journal of sound and vibration, 188(3), p. 385-405.

[21] Ruelle, D., Takens, F., 1971. "On the nature of Turbulence". Communications of Mathematical Physics, 20(3), p. 167-192.

[22] Kounadis A.N. 1992. "On the paradox of the destabilizing effect of damping in non-conservative systems". International Journal of Non-Linear Mechanics, 27, p. 597-609. 